\title{
Synthesis of chiral allenes by peterson-type olefinations
}

Bratovanov, Svetoslav ; Bienz, Stefan

\begin{abstract}
Chiral 1,3-disubstituted allenes have been prepared by means of a Peterson-type elimination in yields as high as $95 \%$. To obtain optically active allenes, the [(benzyloxy)methyl](fert-butyl)methylsilyl group was used as the chiral auxiliary to get an optically enriched elimination precursor. Unfortunately, the elimination reaction with this compound to the respective allene was not stereospecific. Only racemic allene was obtained, probably due to a reaction course via a carbocationic intermediate (Ei rather than E2 mechanism).
\end{abstract}

DOI: https://doi.org/10.1515/MGMC.1996.19.12.769

Posted at the Zurich Open Repository and Archive, University of Zurich

ZORA URL: https://doi.org/10.5167/uzh-141295

Journal Article

Published Version

Originally published at:

Bratovanov, Svetoslav; Bienz, Stefan (1996). Synthesis of chiral allenes by peterson-type olefinations. Main Group Metal Chemistry, 19(12):769.

DOI: https://doi.org/10.1515/MGMC.1996.19.12.769 


\title{
SYNTHESIS OF CHIRAL ALLENES BY PETERSON-TYPE OLEFINATIONS
}

\author{
Svetoslav Bratovanov ${ }^{1}$ ) and Stefan Bienz* \\ Department of Organic Chemistry, University of Zurich, Winterthurerstr. 190, \\ $\mathrm{CH}-8057$ Zurich, Switzerland
}

Abstract

Chiral 1,3-disubstituted allenes have been prepared by means of a Peterson-type elimination in yields as high as $95 \%$. To obtain optically active allenes, the [(benzyloxy)methyl](tert-butyl)methylsilyl group was used as the chiral auxiliary to get an optically enriched elimination precursor. Unfortunately, the elimination reaction with this compound to the respective allene was not stereospecific. Only racemic allene was obtained, probably due to a reaction course via a carbocationic intermediate $\left(E_{1}\right.$ rather than $E_{2}$ mechanism).

\section{Introduction}

The chemistry of allenes has witnessed an immense growth over the past few decades. Allenes not only gained importance as synthetic intermediates for a variety of transformations but they also exhibit interesting and unique physiological properties ${ }^{(1)}$. It is not surprising, therefore, that a rather broad arsenal of methods for the preparation of allenes and cumulenes have been elaborated. Among those, also a Peterson-type of olefination was studied as a means to introduce the two cumulated double bonds ${ }^{(2-4)}$ : $\alpha$-litiated vinylsilanes 1 were coupled with aldehydes to silylsubstituted allyiic alcohols $2(X=O H)$, which were expected to deliver allenes 3 by 1,2-elimination of $\mathrm{R}_{3} \mathrm{SiOH}$ (Scheme 1). However, the treatment of $2(X=O H)$ with base or fluorides resulted only in hydrodesilylation ${ }^{(4,5)}$, and the elimination of $R_{3} S_{1} X(X=0$-acyl or halogen) under the influence of fluorides was found to proceed but sluggishly $(2,3)$. Nevertheless, Torres et al. have used the method for their preparation of an enantiomerically enriched chiral allene, which was obtained, though, not only with poor chemical yield but also with low stereoselectivity $(6)$.
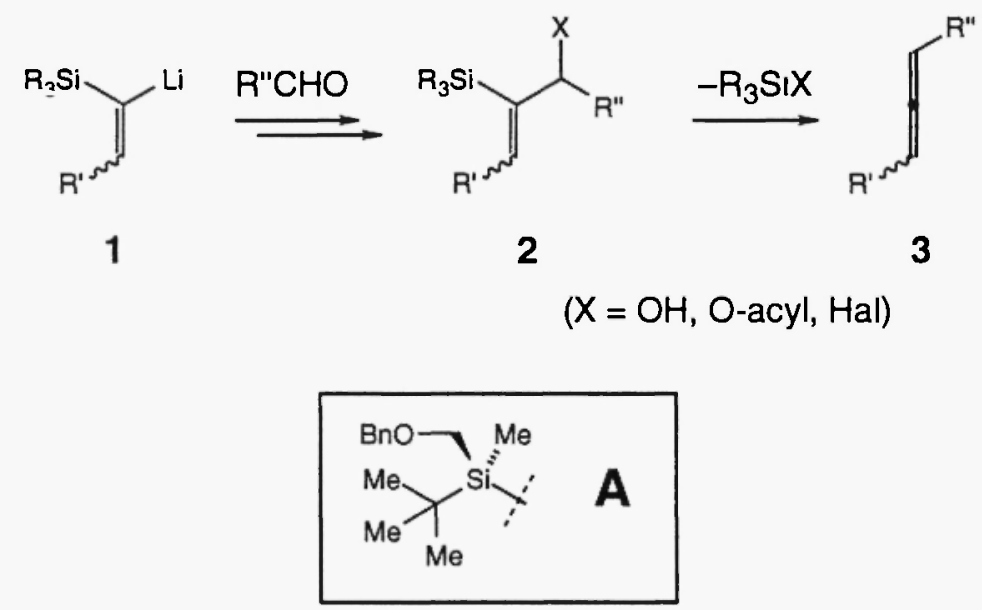

Scheme 1

In connection with our ongoing studies of diastereoselective reactions using the chiral alkoxymethyl-substituted silicon group $\mathbf{A}$ as a stereochemical director ${ }^{(7)}$ and inspired by the results of Torres et al., we became interested in the re-investigation of the above-mentioned Peterson-type

1) Part of the planned PhD thesis of S. B., University of Zurich, Switzerland. 
process. We particularly aimed at an optimization of the reaction conditions for the elimination step-not only to obtain higher yields of the allenes 3 but also in the hope to ensure better stereoselectivities.

\section{Results and Discussion}

The achiral and the racemic silylated alcohols $10 \mathrm{a}-\mathrm{h}$ and $11 \mathrm{a}-\mathrm{h}$ that we used for this study were prepared from the corresponding chlorosilanes 4 and 5 , respectively. Reaction of 4 and 5 with acetylides led to the alkynylsilanes $6 \mathbf{a}, \mathbf{b}$ and $\mathbf{7 a , b}, \mathbf{b}$, which were converted to the vinyl iodides $8 \mathrm{a}-\mathrm{c}$ and $\mathbf{9 a , b}$ by a sequence of hydroalumination and iodination (for $8 \mathrm{c}$ preceded by the deprotonation and benzylation of $6 \mathrm{a}$ to $6 \mathrm{c}$ ). Metal-halogen exchange and reaction with the pertinent aldehydes delivered the desired products 10a-i and 11a-h in good yields (Scheme 2, Table 1). The stereochemistry around the double bonds in the compounds of the type 10 and 11 could be controlled in two ways: by the proper choice of the reaction conditions either in the hydroalumination step or in the metal-halogen exchange. Hydroaluminations of alkynylsilanes with diisobutylaluminum hydride (DIBAH) in hexane are known to give rise to $(E)$-configured $\alpha$-silylalanes, whereas the reactions performed in the presence of donating additives or solvents generally deliver the $(Z)$ configured products ${ }^{(8)}$. In fact, the alkynylsilyl-substituted tert-butyldimethylsiliyl (TBDMS) compounds 6a-c gave-depending solely on the reaction conditions-rise to either of the two possible double bond isomers, as shown in a separate study ${ }^{(9)}$. The reaction of $6 a-c$ with DIBAH in $\mathrm{Et}_{2} \mathrm{O}$ at $-78^{\circ} \mathrm{C}$ followed by quenching with $\mathrm{l}_{2}$ produced the $(E)$-configured iodovinyl silanes $8 \mathrm{a}-\mathrm{C}$; likewise, the corresponding $(Z)$-configured compounds could be obtained when the reactions were performed in hexane. Starting from the chiral alkoxymethyl-substituted silicon compound $7 \mathbf{a}, \mathbf{b}$, on the other hand, only $(E)$-configured vinyl iodides $9 \mathbf{a}, \mathbf{b}$ could be obtained, irrespective of the reaction conditions used. Probably due to internal complexation of the aluminum with the benzyloxymethyl portion of the silicon moiety, the isomerization of the initially formed syn-addition product to the $(E)$-configured $\alpha$-metallated vinylsilane was inhibited.

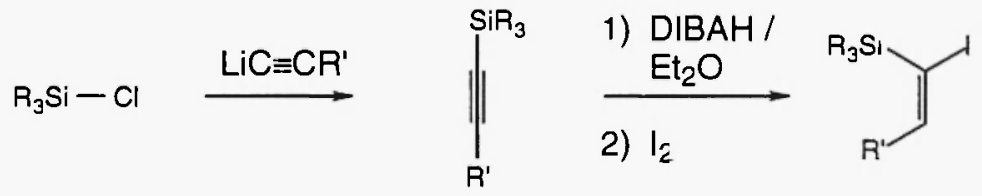

\begin{tabular}{|c|c|c|c|c|c|c|}
\hline $\mathrm{R}_{3} \mathrm{Si}=\mathrm{TBDMS}$ & $\rightarrow$ & $\begin{array}{l}6 a \\
6 b \\
6 c\end{array}$ & $\begin{array}{l}\mathrm{R}^{\prime}=\mathrm{Me} \\
\mathrm{R}^{\prime}=\mathrm{Ph} \\
\mathrm{R}^{\prime}=\mathrm{PhC}_{2} \mathrm{H}_{4}\end{array}$ & $\begin{array}{l}t \text {-BuLi / } \\
\mathrm{BnBr}\end{array}$ & $\begin{array}{l}8 a \\
8 b \\
8 c\end{array}$ & $\begin{array}{l}\mathrm{R}^{\prime}=\mathrm{Me} \\
\mathrm{R}^{\prime}=\mathrm{Ph} \\
\mathrm{R}^{\prime}=\mathrm{PhC}_{2} \mathrm{H}_{4}\end{array}$ \\
\hline $\mathrm{R}_{3} \mathrm{Si}=\mathbf{A}$ & & $\begin{array}{l}7 a \\
7 b\end{array}$ & $\begin{array}{l}R^{\prime}=\mathrm{Me} \\
R^{\prime}=\mathrm{Ph}\end{array}$ & & $\begin{array}{l}9 a \\
9 b\end{array}$ & $\begin{array}{l}R^{\prime}=M e \\
R^{\prime}=P h\end{array}$ \\
\hline
\end{tabular}

1) BuLi / $\mathrm{Et}_{2} \mathrm{O}$ or Hexane

2) $\mathrm{R} " \mathrm{CHO}$

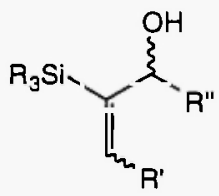

$10 a-i / 11 a-h$

(see Table 1)

Scheme 2

Nevertheless, for both types of silanes $\left(\mathrm{R}_{3} \mathrm{Si}=\right.$ TBDMS or $\left.\mathbf{A}\right)$ both double bond isomers of the silylated allylic alcohols of the types 10 and 11 could be attained. In accordance with published results $(10,11)$, we found that the $(Z)$-configured $\alpha$-silylated vinyllithium intermediates, obtained from 
Table 1: Preparation of the Silyl-Substituted Allylic Alcohols 10a-i and 11a-h.

\begin{tabular}{|c|c|c|c|c|c|c|c|}
\hline \multirow[t]{2}{*}{ Entry } & \multirow{2}{*}{$\begin{array}{l}\text { Educt } \\
\text { No }\end{array}$} & \multicolumn{6}{|c|}{ Product } \\
\hline & & No & $\mathrm{R}_{3} \mathrm{Si}$ & $\mathrm{R}^{\prime}$ & $R^{\prime \prime \prime}$ & $\begin{array}{l}\text { Double } \\
\text { Bond }\end{array}$ & $\begin{array}{l}\text { Yield } \\
(\%)\end{array}$ \\
\hline 1 & $8 a$ & $10 a$ & TBDMS & $\mathrm{Me}$ & $\mathrm{Ph}$ & $Z$ & 81 \\
\hline 2 & $8 a$ & $10 \mathrm{~b}$ & TBDMS & $\mathrm{Me}$ & $\mathrm{Ph}$ & $E$ & 74 \\
\hline 3 & $8 \mathrm{~b}$ & $\begin{array}{l}10 \mathrm{c} \\
10 \mathrm{~d}\end{array}$ & $\begin{array}{l}\text { TBDMS } \\
\text { TBDMS }\end{array}$ & $\begin{array}{l}\mathrm{Ph} \\
\mathrm{Ph}\end{array}$ & $\begin{array}{l}\text { Et } \\
\text { Et }\end{array}$ & $\begin{array}{l}Z \\
E\end{array}$ & $\left.87(10: 1)^{a}\right)$ \\
\hline 4 & $8 \mathrm{~b}$ & $10 \mathrm{e}$ & TBDMS & $\mathrm{Ph}$ & $\mathrm{Ph}$ & $z$ & 82 \\
\hline 5 & $8 b$ & $10 f$ & TBDMS & $\mathrm{Ph}$ & $\mathrm{Ph}$ & $E$ & 79 \\
\hline 6 & $8 b$ & $10 \mathrm{~g}$ & TBDMS & $\mathrm{Ph}$ & $\mathrm{i}-\mathrm{Pr}$ & $Z$ & 82 \\
\hline 7 & $8 \mathrm{c}$ & $\begin{array}{l}10 \mathrm{~h} \\
10 \mathrm{i}\end{array}$ & $\begin{array}{l}\text { TBDMS } \\
\text { TBDMS }\end{array}$ & $\begin{array}{l}\mathrm{PhC}_{2} \mathrm{H}_{4} \\
\mathrm{PhC}_{2} \mathrm{H}_{4}\end{array}$ & $\begin{array}{l}\mathrm{Ph} \\
\mathrm{Ph}\end{array}$ & $\begin{array}{l}Z \\
E\end{array}$ & $\left.83(15: 1)^{a}\right)$ \\
\hline 8 & $9 a$ & $\begin{array}{l}11 a \\
11 b\end{array}$ & $\begin{array}{l}A \\
A\end{array}$ & $\begin{array}{l}\mathrm{Me} \\
\mathrm{Me}\end{array}$ & $\begin{array}{l}\mathrm{Ph} \\
\mathrm{Ph}\end{array}$ & $\begin{array}{l}Z \\
Z\end{array}$ & $\left.69(2: 1)^{a}\right)$ \\
\hline 9 & $9 a$ & $\begin{array}{l}11 \mathrm{c} \\
11 \mathrm{~d}\end{array}$ & $\begin{array}{l}\mathbf{A} \\
\mathbf{A}\end{array}$ & $\begin{array}{l}\mathrm{Me} \\
\mathrm{Me}\end{array}$ & $\begin{array}{l}\mathrm{Ph} \\
\mathrm{Ph}\end{array}$ & $\begin{array}{l}E \\
E\end{array}$ & $\left.60(5: 7)^{a}\right)$ \\
\hline 10 & $9 a$ & $\begin{array}{l}11 \mathrm{e} \\
11 \mathrm{f}\end{array}$ & $\begin{array}{l}\mathbf{A} \\
\mathbf{A}\end{array}$ & $\begin{array}{l}\mathrm{Me} \\
\mathrm{Me}\end{array}$ & $\begin{array}{l}\mathrm{i}-\mathrm{Pr} \\
\mathrm{i}-\mathrm{Pr}\end{array}$ & $\begin{array}{l}Z \\
Z .\end{array}$ & $\left.93(1: 2)^{a}\right)$ \\
\hline 11 & $9 \mathrm{~b}$ & $\begin{array}{l}11 \mathrm{~g} \\
11 \mathrm{~h} \\
\end{array}$ & $\begin{array}{l}\mathbf{A} \\
\mathbf{A}\end{array}$ & $\begin{array}{l}\mathrm{Ph} \\
\mathrm{Ph}\end{array}$ & $\begin{array}{l}\mathrm{Ph} \\
\mathrm{Ph}\end{array}$ & $\begin{array}{l}E \\
E\end{array}$ & $\left.80(5: 6)^{a}\right)$ \\
\hline
\end{tabular}

the respective $(E)$-configured vinyl iodides, can be isomerized to the more stable $(E)$-configured analogs. The rate of isomerization of the $\alpha$-metallated vinyl silicon species depends crucially on the reaction temperature and the solvent: generating and keeping the vinyllithium species in hexane at $-80^{\circ} \mathrm{C}$ (or below) allowed to retain their double bond geometries; warming them up to $-5^{\circ} \mathrm{C}$ or holding them in a donating solvent like $\mathrm{Et}_{2} \mathrm{O}$ or THF for a few hours, resulted in partial or complete double bond inversion. Thus, in general both double bond isomers of the compounds of the type 10 and 11 could be prepared from the corresponding common $(E)$-configured vinyl iodides of the type 8 or 9 by the proper choice of the reaction conditions (see Exp. Parf). A problem, though, arose still in the preparation of the (Z)-configured alkoxymethyl-substituted silicon compounds of the type 11: since these compounds possess internally a donating ether functionality, it was rather arduous to retain the doble bond geometry of the respective $(Z)$-configured vinyllithium species after the metal-halogen exchange; the reaction temperature had to be strictly kept below $-90^{\circ} \mathrm{C}$ to minimize double bond inversion. Even this was not sufficient for the reaction with the phenyl-substituted derivative $9 b$, whose corresponding lithiated species isomerized especially readily. For this starting material, the double bond geometry could be retained to only $30 \%$ at its best.

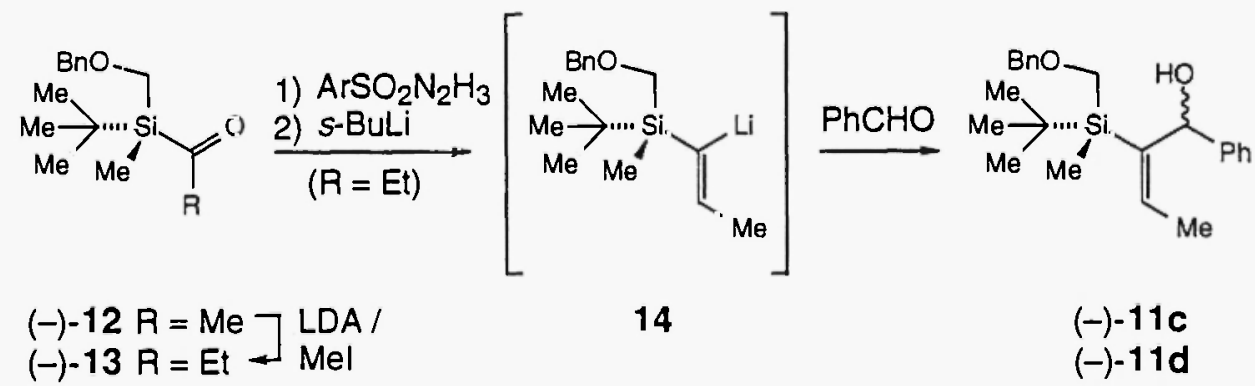

Scheme 3

Optically active $11 \mathrm{c}, \mathrm{d}$ were obtained by a different route (Scheme 3 ). Enantiomerically enriched acetylsilane $(-)-12(>96 \% \mathrm{ee})^{(12)}$ was alkylated with methyl iodide via the lithium enolate, and the resulting ethyl silyl ketone $(-)-13$ was converted to the corresponding $\alpha$-lithiated vinyl- 
silane 14 by the Shapiro reaction. Addition of the organolithium species to benzaldehyde afforded the two alcohols $(-)-11 \mathrm{c}$ and $(-)-11 \mathrm{~d}$. The stereoselectivity in the addition reaction proved to be disappointingly low (dr ca. 1.2:1). However, oxidation of the chiral alcohols of the type 11 to the corresponding ketones, followed by re-reduction with $\mathrm{LiAlH}_{4}$, produced one of the respective alcohols with virtually complete stereoselection.<smiles>[R]/C=C(/[Ga])[C@H](O)[18OH]</smiles>

$10 \mathrm{a}-\mathrm{h} / 11 \mathrm{a}, \mathrm{c}, \mathrm{e}$

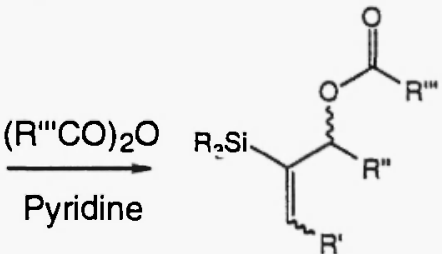

$15 a-m / 16 a-c$ (see Table 2)

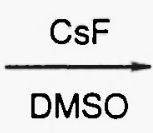<smiles>CC=CCBr</smiles>

$17 a-e$

(see Table 2)

Scheme 4

Allene formation was optimized with several 0-acyl compounds of the type 15 and 16 prepared from the parent alcohols of the type 10 and 11 (Scheme 4 and Table 2). Trifluoroacetates as $15 \mathrm{a}\left(\mathrm{R}^{\prime \prime \prime}=\mathrm{F}_{3} \mathrm{C}\right)$, which were used in preceding investigations $(2,3,6)$, proved to be tedious to access, and their reaction with several fluorides did not provide any allenés. Instead, as shown exemplary with the transformation of 15a, the reaction with, e.g., CsF in DMSO gave rise to negliable amounts of allene 17a; the major products being the 'hydrolysis' compounds 11a and 11b (!).

Table 2: Conversion of the Silyl-Substituted Allylic Alcohols 10a-h and 11a,c,e to the Allenes $17 a-e$.

\begin{tabular}{|c|c|c|c|c|c|c|c|c|c|c|}
\hline \multirow[t]{2}{*}{ Entry } & \multicolumn{6}{|l|}{ Educt } & \multicolumn{2}{|c|}{ Conditions } & \multicolumn{2}{|c|}{ Product } \\
\hline & No & $\mathrm{R}_{3} \mathrm{Si}$ & $\mathrm{R}^{\prime}$ & $\mathrm{R}^{\prime \prime}$ & $\begin{array}{l}\text { Double } \\
\text { Bond }\end{array}$ & $\mathrm{R}^{\prime \prime \prime}$ & $\mathrm{T}\left({ }^{\circ} \mathrm{C}\right)$ & $t(h)$ & No & $\begin{array}{l}\text { Yield } \\
(\%)\end{array}$ \\
\hline 1 & $15 a$ & TBDMS & $\mathrm{Me}$ & $\mathrm{Ph}$ & $Z$ & $\mathrm{~F}_{3} \mathrm{C}$ & 23 & 3 & $17 a$ & 2 \\
\hline 2 & $15 b$ & TBDMS & $\mathrm{Me}$ & $\mathrm{Ph}$ & $Z$ & $\mathrm{Me}$ & 140 & 6 & $17 a$ & 55 \\
\hline 3 & $15 c$ & TBDMS & $\mathrm{Me}$ & $\mathrm{Ph}$ & $Z$ & $\mathrm{H}_{2} \mathrm{ClC}$ & 75 & 48 & $17 a$ & 31 \\
\hline 4 & $15 c$ & TBDMS & $\mathrm{Me}$ & $\mathrm{Ph}$ & $Z$ & $\mathrm{H}_{2} \mathrm{CiC}$ & 100 & 8 & $17 a$ & 33 \\
\hline 5 & $15 \mathrm{c}$ & TBDMS & $\mathrm{Me}$ & $\mathrm{Ph}$ & $\bar{Z}$ & $\mathrm{H}_{2} \mathrm{ClC}$ & 140 & 1.25 & $17 a$ & 50 \\
\hline 6 & $15 d$ & TBDMS & $\mathrm{Me}$ & $\mathrm{Ph}$ & $E$ & $\mathrm{Me}$ & 120 & 96 & $17 a$ & 30 \\
\hline 7 & $15 e$ & TBDMS & $\mathrm{Ph}$ & Et & $\bar{Z}$ & $\mathrm{Me}$ & 100 & 2.5 & $17 b$ & 95 \\
\hline 8 & $15 t$ & TBDMS & $\mathrm{Ph}$ & Et & $Z$ & $\mathrm{H}_{2} \mathrm{ClC}$ & 100 & 1.5 & $17 b$ & 82 \\
\hline 9 & $15 \mathrm{~g}$ & TBDMS & $\mathrm{Ph}$ & Et & $z$ & $\mathrm{HCi}_{2} \mathrm{C}$ & 100 & 0.8 & $17 b$ & 64 \\
\hline 10 & $15 \mathrm{~h}$ & TBDMS & $\mathrm{Ph}$ & Et & $E$ & $\mathrm{H}_{2} \mathrm{ClC}$ & 100 & 21 & $17 b$ & 57 \\
\hline 11 & $15 i$ & TBDMS & $\mathrm{Ph}$ & $\mathrm{Ph}$ & $z$ & $\mathrm{Me}$ & 120 & 1 & $17 c$ & 71 \\
\hline 12 & $15 j$ & TBDMS & $\mathrm{Ph}$ & $\mathrm{Ph}$ & $E$ & $\mathrm{Me}$ & 120 & 96 & $17 \mathrm{c}$ & 25 \\
\hline 13. & $15 k$ & TBDMS & $\mathrm{Ph}$ & $\mathrm{i}-\mathrm{Pr}$ & $Z$ & $\mathrm{HCl}_{2} \mathrm{C}$ & 100 & 120 & $17 d$ & $<2$ \\
\hline 14 & 151 & TBDMS & $\mathrm{PhC}_{2} \mathrm{H}_{4}$ & $\mathrm{Ph}$ & $Z$ & $\mathrm{Me}$ & 120 & 10 & $17 \mathrm{e}$ & 71 \\
\hline 15 & $15 \mathrm{~m}$ & TBDMS & $\mathrm{PhC}_{2} \mathrm{H}_{4}$ & $\mathrm{Ph}$ & $E$ & $\mathrm{H}_{2} \mathrm{ClC}$ & 120 & 2 & $17 \mathrm{e}$ & 60 \\
\hline 16 & $16 a$ & A & $\mathrm{Me}$ & $\mathrm{Ph}$ & $Z$ & $\mathrm{Me}$ & 100 & 24 & $17 a$ & 50 \\
\hline 17 & $16 b$ & A & $\mathrm{Me}$ & $\mathrm{Ph}$ & $E$ & $\mathrm{Me}$ & 120 & $\left.48^{a}\right)$ & $17 a$ & 20 \\
\hline 18 & $16 \mathrm{c}$ & A & $\mathrm{Me}$ & i-Pr & $Z$ & $\mathrm{Me}$ & 120 & $\left.48^{b}\right)$ & $17 d$ & $\left.0^{b}\right)$ \\
\hline
\end{tabular}

a) Not complete conversion; $\left.{ }^{b}\right)$ No reaction.

The formation of allene was successfully realized with compounds of the type 15 and 16 that possess less potent leaving groups. Since allenes decompose readily upon prolonged heating, however, the choice of the proper reaction conditions to secure a rapid conversion the acyl 
compounds was crucial. Best results were obtained when the least-activated acetates of the type 15 or 16 possessing the (Z)-configured double bond (Entries $2,7,11,14$, and 16, Table 2) were treated with fluorides in DMSO at $100-140^{\circ} \mathrm{C}$ for several hours. Yields of up to $95 \%$ (Entry 7, Table 2) of the parent allenes were gained, which is a remarkable improvement as compared to earlier attempts that delivered allenes with $60 \%$ yield at their best ${ }^{(2,3)}$. Though the reaction rates were higher with starting compounds possessing more activated groups (cf. reactions of $15 \mathrm{c}$, Entries 35 , Table 2), the chloro- and dichloroacetates gave still lower yields of the desired allenes due to more side reactions.

Much to our disappointment, the fluoride treatment of optically active silane $(-)-16 \mathrm{~b}$ gave the corresponding allene 17a in low yields and as a racemate only (Entry 17, Table 2). The earlier results of Torres et al. ${ }^{(6)}$, who obtained the same product in $18 \%$ ee (assumingly by an antielimination), could not be verified with our compound. Supposedly, the formation of the allenes proceeds mainly by an $E_{1}$ rather than an $E_{2}$ mechanism. The $E_{1}$ mechanism is probably particularly favored for the $(E)$-configured starting materials, where the transition states for concerted $E_{2}$ elimination processes are strongly disfavored due to large $A_{1,3}$ strain. The $E_{2}$ mechanism could, however, still be preferred for the $(Z)$-configured elimination precursors. The reaction course by an $E_{2}$ mechanism can be the explanation for the fact that the (Z)-configured silylated alkenes 15 are converted markedly faster to the corresponding allenes of the type 17 as compared to the $(E)$-configured counterparts (cf., e.g., Entries 5/6, 11/12, 14/15, and 16/17). Since optically active (Z)-configured acetates of the type 16 are not accessible, however, this hypothesis cannot be proven momentarily.

Acknowledgement: We thank the members of our analytical laboratories for their excellent services and the Swiss National Science Foundation for their generous financial support. We are especially grateful to Prof. Dr. M. Hesse who provided us with laboratory space, equipment, and regular occasions for scientific discussions.

\section{Experimental Part}

General. Unless otherwise stated: all organic solvents were distilled prior to use. For the reactions $\mathrm{THF}$ and $\mathrm{Et}_{2} \mathrm{O}$ were dried over $\mathrm{Na} /$ ketyl. All reactions were carried out under a blanket of $\mathrm{Ar}$. Soln. for workup procedures were prepared in deionized $\mathrm{H}_{2} \mathrm{O}$. Chromatography: silica gel Merck 60 (40-63 $\mu \mathrm{m})$. M.p.: Mettler FP-5/FP-52. IR Spectra: Perkin-Elmer 781; as films between NaCl plates; data in $\mathrm{cm}^{-1}$. ${ }_{1} \mathrm{H}$ NMR: at $300 \mathrm{MHz}$ in $\mathrm{CDCl}_{3}$; Bruker $A C-300, \delta$ in ppm rel. to $\mathrm{CHCl}_{3}(\delta=7.26), J$ in $\mathrm{Hz}$. ${ }^{13} \mathrm{C} \mathrm{NMR}$ : at $75.6 \mathrm{MHz}$ in $\mathrm{CDCl}_{3}$; Bruker $\mathrm{ARX}-300 ; \delta$ in ppm rel. to $\mathrm{CHCl}_{3}(\delta=77.0)$, multiplicities from DEPT-135 and DEPT-90 experiments; Cl-MS (chemical-ionization mass spectrometry): Finnigan MAT SSQ 700 or Varian MAT 711i; reactant gas: $\mathrm{NH}_{3}$; data in $\mathrm{m} / \mathrm{z}$.

1. Preparation of Alkynylsilanes. - 1.1. (tert-Butyl)dimethyl(prop-1-ynyl)silane (6a). Propyne was passed through a solution of BuLi (10 ml, $2 \mathrm{~N}$ in pentane) in hexane (10 ml) at $-78^{\circ} \mathrm{C}$ until the solution turned into a white gel-like solid that was dissolved by addition of THF $(10 \mathrm{ml})$. It was cooled to $-78^{\circ} \mathrm{C}$ and tert-butyl(chloro)dimethylsilane $(4,2.13 \mathrm{~g}, 14.13 \mathrm{mmol}$, dissolved in $10 \mathrm{ml}$ of THF) was added dropwise. The temperature was slowly raised to $23^{\circ} \mathrm{C}(2 \mathrm{~h})$, and the solution was stirred for an additional $16 \mathrm{~h}$. It was re-cooled to $-50^{\circ} \mathrm{C}$ and quenched with $10 \%$ aqueous $\mathrm{HCl}$ solution The aqueous layer was extracted with $\mathrm{Et}_{2} \mathrm{O}$, and the combined organic phases were dried over $\mathrm{MgSO}_{4}$ and evaporated. The crude product gave after distillation (bulb-to-bulb, $70^{\circ} \mathrm{C} / 10^{-3}$ torr) $6 \mathrm{a}\left(1.67 \mathrm{~g}, 10.84 \mathrm{mmol}, 70 \%\right.$ ) as a colorless oil. IR $\left(\mathrm{CHCl}_{3}\right): 3000 \mathrm{w}, 2950 \mathrm{~s}, 2930 \mathrm{~s}, 2890 \mathrm{~m}$, $2860 \mathrm{~s}, 2180 \mathrm{~s}, 1470 \mathrm{~m}, 1460 \mathrm{~m}, 1410 \mathrm{w}, 1390 \mathrm{w}, 1360 \mathrm{w}, 1250 \mathrm{~s}, 1025 \mathrm{~s}, 1010 \mathrm{~m}, 940 \mathrm{w}, 840 \mathrm{~s}, 820 \mathrm{~s}$,

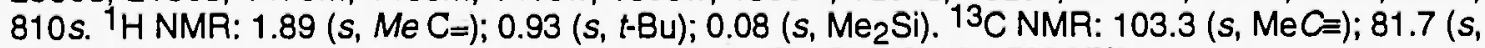
$\mathrm{SiC} \equiv) ; 26.0\left(q, \mathrm{Me}_{3} \mathrm{C}\right) ; 16.4\left(s, \mathrm{Me}_{3} \mathrm{C}\right) ;-4.6\left(q, \mathrm{Me}_{2} \mathrm{Si}\right)$. Cl-MS: $155\left([\mathrm{M}+\mathrm{H}]^{+}\right)$.

1.2. (tert-Butyl)dimethyl(2-phenylethynyl)silane (6b). Analogeously to 6a, phenyl acetylene $(5.8 \mathrm{ml}, 50 \mathrm{mmol})$, deprotonated with BuLi $(60 \mathrm{mmol})$ and reacted with $4(5.0 \mathrm{~g}, 40.9 \mathrm{mmol})$, gave after distillation (bulb-to-bulb, $100^{\circ} \mathrm{C} / 10^{-4}$ torr) $6 \mathrm{~b}(5.9 \mathrm{~g}, 27.5 \mathrm{mmol}, 82 \%)$ as a colorless oil. IR $\left(\mathrm{CHCl}_{3}\right): 3300 w, 3080 w, 3060 w, 3000 w, 2950 s, 2920 s, 2880 s, 2850 s, 2150 s, 1590 w, 1490 s$, $1470 \mathrm{~s}, 1460 \mathrm{~s}, 1440 \mathrm{~m}, 1410 \mathrm{w}, 1390 \mathrm{w}, 1360 \mathrm{w}, 1250 \mathrm{~s}, 1070 \mathrm{w}, 1025 \mathrm{w}, 1005 \mathrm{~m}, 940 \mathrm{w}, 915 \mathrm{w}$, 840s. 1H NMR: 7.49-7.29 (m, 5 arom. $\mathrm{H}) ; 1.01$ ( $s, t-\mathrm{Bu}) ; 0.19$ ( $\left.s, \mathrm{Me}_{2} \mathrm{Si}\right) .{ }^{13} \mathrm{C}$ NMR: 132.0 (d, 2 
arom. C); 128.3 (d, arom. C); 128.2 (d, 2 arom. C); 123.2 (s, arom. C); 105.7 (s, PhCझ); 92.4 (s, $\mathrm{SiC}=) ; 26.1\left(q, \mathrm{Me}_{3} \mathrm{C}\right) ; 16.6\left(\mathrm{~s}, \mathrm{Me}_{3} \mathrm{C}\right) ;-4.7$ (q, Mes $\left.\mathrm{Si}\right) . \mathrm{Cl}-\mathrm{MS}: 217\left([\mathrm{M}+\mathrm{H}]^{+}\right)$.

1.3. (tert-Butyl)dimethyl(4-phenylbut-1-ynyl)silane (6c). To a solution of $6 \mathrm{a}(0.30 \mathrm{~g}, 1.95$ $\mathrm{mmol})$ in THF $(8 \mathrm{ml})$ was added BuLi $(1.5 \mathrm{ml}, 2 \mathrm{~N}$ in pentane $)$ at $-80^{\circ} \mathrm{C}$. After $1 \mathrm{~h}$, a solution of benzyl bromide $(0.43 \mathrm{mg}$ dissolved in $2 \mathrm{ml}$ of THF) was added dropwise. The temperature was gradually raised to $-60^{\circ} \mathrm{C}(10 \mathrm{~min})$. It was stirred for an additional $1 \mathrm{~h}$ and quenched at $-80^{\circ} \mathrm{C}$ with saturated aqueous $\mathrm{NH}_{4} \mathrm{Cl}$ solution $(10 \mathrm{ml})$. The aqueous layer was extracted with $\mathrm{Et}_{2} \mathrm{O}$, and the combined organic phases were dried over $\mathrm{MgSO}_{4}$ and evaporated. The crude product gave after chromatography (hexane) $6 c(0.38 \mathrm{~g}, 1.56 \mathrm{mmol}, 80 \%)$ as a colorless oil. IR $\left(\mathrm{CHCl}_{3}\right): 3080 \mathrm{w}, 3060 \mathrm{w}, 3020 \mathrm{~m}$, $2950 \mathrm{~s}, 2925 \mathrm{~s}, 2900 \mathrm{~m}, 2835 \mathrm{~s}, 2180 \mathrm{~s}, 1600 \mathrm{w}, 1490 \mathrm{~m}, 1470 \mathrm{~m}, 1460 \mathrm{~m}, 1450 \mathrm{~m}, 1425 \mathrm{w}, 1410 \mathrm{w}$, $1375 w, 1360 m, 1335 w, 1250 s, 1120 w, 1095 w, 1075 w, 1040 m, 1030 m, 1005 m, 995 w, 940 w$, $905 w, 870 m, 835 s, 825 s, 810 s, 775 s, 745 m, 695 s, 680 m, 620 m$. ${ }^{1}$ H NMR: $7.29-7.15(m, 5$ arom. $\mathrm{H}) ; 2.87\left(t, J=7.6, \mathrm{PhCH}_{2}\right) ; 2.50\left(t, \mathrm{CH}_{2} \mathrm{C} \equiv\right) ; 0.88(s, t-\mathrm{Bu}) ; 0.03\left(\mathrm{~s}, \mathrm{Me}_{2} \mathrm{Si}\right) .{ }^{13} \mathrm{C}$ NMR: $140.5(s$, arom. C); 128.4, 128.2 (2d, each 2 arom. C); 126.1 (d, arom. C); $107.0\left(\mathrm{~s}, \mathrm{CH}_{2} \mathrm{C}=\right)$; 83.3 (s, $\mathrm{SiC}=$ ); $35.1\left(t, \mathrm{PhCH}_{2}\right) ; 26.0$ (q, Mes $\left.\mathrm{C}\right) ; 22.0\left(t, \mathrm{CH}_{2} \mathrm{C}=\right) ; 16.4\left(s, \mathrm{Me}_{3} \mathrm{C}\right) ;-4.6$ (q, Me $\left.2 \mathrm{Si}\right) . \mathrm{Cl}-\mathrm{MS}: 262$ $\left(\left[\mathrm{M}+\mathrm{NH}_{4}\right]^{+}\right)$.

1.4. [(Benzyloxy)methyl](tert-butyl)methyl(prop-1-ynyl)silane (7a). Analogeously to 6a, propyne, deprotonated with BuLi $(6.0 \mathrm{mmol})$ and reacted with [(benzyloxy)methyl](tert-butyl)(chloro)dimethylsilane $(5,5.0 \mathrm{~g}, 40.9 \mathrm{mmol})$, gave after filtration through a plug of silica gel (toluene) $7 \mathrm{a}$ $(0.88 \mathrm{~g}, 35.2 \mathrm{mmol}, 86 \%)$ as a colorless oil. The product was pure as determined by NMR spectroscopy and used in the next reaction without further purification. IR $\left(\mathrm{CHCl}_{3}\right): 3080 \mathrm{w}, 3060 \mathrm{w}, 3020 \mathrm{w}$, $2950 s, 2922 s, 2890 m, 2850 s, 2810 w, 2180 s, 1495 w, 1470 m, 1460 m, 1450 m, 1390 w, 1380 m$, $1360 \mathrm{~m}, 1250 \mathrm{~m}, 1205 \mathrm{~m}, 1090 \mathrm{~s}, 1072 \mathrm{~s}, 1028 \mathrm{~s}, 1010 \mathrm{w}, 980 \mathrm{w}, 940 \mathrm{w}, 905 \mathrm{w}, 830 \mathrm{~s}, 785 \mathrm{~s}, 762 \mathrm{~s}$, $730 \mathrm{~s}, 695 \mathrm{~s}$. ${ }^{\mathrm{H}} \mathrm{H}$ NMR: $7.32-7.24(\mathrm{~m}, 5 \mathrm{arom}$. $\mathrm{H}) ; 4.45\left(\mathrm{~s}, \mathrm{PhCH}_{2} \mathrm{O}\right) ; 3.23,3.17(A B, J=12.9$, $\mathrm{SiCH}_{2} \mathrm{O}$ ); $1.84(s, \mathrm{MeC}=) ; 0.91(s, t-\mathrm{Bu}) ; 0.10$ ( $\left.s, \mathrm{MeSi}\right)$. ${ }^{3} \mathrm{C}$ NMR: 139.1 ( $s$, arom. C); 128.45, 127.8 (2d, each 2 arom. C); 127.7 (d, arom. C); $125.6(s, \mathrm{MeC}=) ; 104.8(\mathrm{~s}, \mathrm{SiC}=) ; 77.1(t, \mathrm{PhCH} 2 \mathrm{O}$ ); $61.32\left(t, \mathrm{SiCH}_{2} \mathrm{O}\right) 26.7\left(q, \mathrm{Me}_{3} \mathrm{C}\right) ; 26.4(q, \mathrm{MeC}=) ; 17.0$ (s, Mes $\left.\mathrm{C}\right) ; 5.2$ (q, MeSi). Cl-MS: 278 $\left(\left[\mathrm{M}+\mathrm{NH}_{4}\right]^{+}\right)$.

1.5. [(Benzyloxy)methyl](tert-butyl)methyl(2-phenylethynyl)silane (7b). Analogeously to $6 \mathrm{a}$, phenyl acetylene $(1.54 \mathrm{ml}, 14.1 \mathrm{mmol})$, deprotonated with BuLi $(14.8 \mathrm{mmol})$ and reacted with 5 $(1.95 \mathrm{~g}, 7.60 \mathrm{mmol})$, gave after chromatography (hexane $\left./ \mathrm{CH}_{2} \mathrm{Cl}_{2} 1: 1\right) 7 \mathrm{~b}(2.42 \mathrm{~g}, 7.52 \mathrm{mmol}, 98 \%)$ as a colorless oil. IR: $3080 w, 3060 w, 3030 m, 2950 s, 2930 s, 2890 s, 2850 s, 2810 m, 2740 w$, $2710 w, 2160 s, 1595 w, 1570 w, 1490 m, 1470 m, 1460 m, 1440 m, 1430 w, 1390 w, 1380 m, 1360 m$, $1300 w, 1250 \mathrm{~m}, 1220 \mathrm{~m}, 1175 \mathrm{w}, 1155 \mathrm{w}, 1090 \mathrm{~s}, 1070 \mathrm{~s}, 1025 \mathrm{~m}, 1010 \mathrm{~m}, 980 \mathrm{w}, 940 \mathrm{w}, 965 \mathrm{w}$, $955 w, 835 s, 800 m, 780 s, 755 s, 735 s, 720 m, 690 s$. ${ }^{1 H}$ NMR: 7.53-7.21 (m, 10 arom. H); 4.51(s,

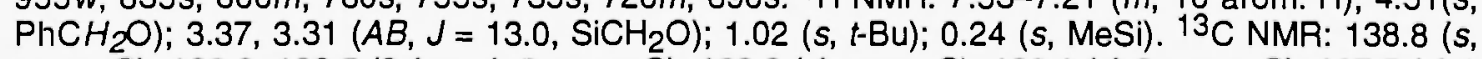
arom. C); $132.0,128.5$ (2d, each 2 arom. C); 128.2 (d, arom. C); 128.1 (d, 2 arom. C); 127.5 (d, 3 arom. C); 123.0 (s, arom. C); 106.8 (s, PhC $\equiv) ; 90.1$ (s, SiC $\equiv) ; 76.8\left(t, \mathrm{PhCH}_{2} \mathrm{O}\right) ; 60.9$ (t, $\left.\mathrm{SiCH}_{2} \mathrm{O}\right)$; 26.5 (q, Mes $\mathrm{C}) ; 19.9\left(\mathrm{~s}, \mathrm{Me}_{3} \mathrm{C}\right) ;-7.5$ (q, MeSi). Cl-MS: $323\left([\mathrm{M}+\mathrm{H}]^{+}\right)$.

2. Preparation of 1-lodoviny/silanes. - 2.1. (E)-(tert-Butyl)(1-iodoprop-1-enyl)dimethylsilane (8a). To a solution of $6 \mathrm{a}(2.0 \mathrm{~g}, 14.0 \mathrm{mmol})$ in $\mathrm{Et}_{2} \mathrm{O}(30 \mathrm{ml})$ was slowly added diisobutylaluminum hydride (DIBAH, $13 \mathrm{ml}, 20 \%$ in toluene) at $23^{\circ} \mathrm{C}$. The mixture was refluxed for $8 \mathrm{~h}$, cooled to $-50^{\circ} \mathrm{C}$, and dropwise treated with an iodine solution $(3.96 \mathrm{~g}, 15.58 \mathrm{mmol}$, dissolved in $7 \mathrm{ml}$ of THF). The mixture was allowed to warm to $23^{\circ} \mathrm{C}$, and stirring was continued for $2 \mathrm{~h}$ before it was quenched with a saturated aqueous $\mathrm{NH}_{4} \mathrm{Cl}$ solution $(20 \mathrm{ml})$ at $-50^{\circ} \mathrm{C}$. The aqueous layer was extracted with $\mathrm{Et}_{2} \mathrm{O}$, and the combined organic pahses were dried over $\mathrm{MgSO}_{4}$ and evaporated. The crude product gave after distillation (bulb-to-bulb, $100^{\circ} \mathrm{C} / 10^{-4}$ torr) $8 \mathrm{a}(2.49 \mathrm{~g}, 8.86 \mathrm{mmol}$, $68 \%$ as a slightly yellow oil: IR $\left(\mathrm{CHCl}_{3}\right): 3000 \mathrm{~m}, 2960 \mathrm{~s}, 2930 \mathrm{~s}, 2890 \mathrm{~s}, 2860 \mathrm{~s}, 1590 \mathrm{w} .1470 \mathrm{~m}$, $1465 m, 1445 w, 1405 w, 1390 w, 1360 w, 1325 w, 1250 s, 1110 w, 1050 w, 987 w, 840 s$. iH NMR: 7.42 ( $q, \mathrm{HC}=) ; 1.72$ (d, MeHC=); 0.99 (s, $t$-Bu); 0.31 (s, Mez2Si). ${ }^{13} \mathrm{C}$ NMR: 153.9 (d, HC=); 104.3 (s, $\mathrm{SiC}=) ; 28.3\left(q, \mathrm{Me}_{3} \mathrm{C}\right) ; 22.9\left(\mathrm{~s}, \mathrm{Me}_{3} \mathrm{C}\right) ; 0.0\left(q, \mathrm{Me}_{2} \mathrm{Si}\right)$. Cl-MS: $283\left([\mathrm{M}+\mathrm{H}]^{+}\right)$.

2.2. (E')-(tert-Butyl)(1-iodo-2-phenylethenyl)dimethylsilane (8b). Analogeously to $8 \mathrm{a}, 6 \mathrm{~b}$ $(3.0 \mathrm{~g}, 14.0 \mathrm{mmol})$, reacted with DIBAH $(17.3 \mathrm{ml})$ and iodine $(5.29 \mathrm{~g}, 20.83 \mathrm{mmol})$, gave after distillation (bulb-to-bulb, $100^{\circ} \mathrm{C} / 10^{-4}$ torr) $8 \mathrm{~b}(3.61 \mathrm{~g}, 10.52 \mathrm{mmol}, 75 \%)$ as a slightly yellow oil. IR $\left(\mathrm{CDCl}_{3}\right): 3000 \mathrm{~m}, 2960 \mathrm{~s}, 2930 \mathrm{~s}, 2890 \mathrm{~s}, 2860 \mathrm{~s}, 2160 \mathrm{w}, 1600 \mathrm{w}, 1565 \mathrm{w}, 1490 \mathrm{~m}, 1470 \mathrm{~s}, 1465 \mathrm{~s}$, $1445 \mathrm{~m}, 1410 w, 1395 w, 1365 \mathrm{~m}, 1255 \mathrm{~s}, 1100 w, 1070 w, 1010 \mathrm{~m}, 990 w, 940 w, 930 w, 840 \mathrm{~s}, 820 \mathrm{~s}$, 810s. ${ }^{1} \mathrm{H}$ NMR: $8.54(\mathrm{~s}, \mathrm{HC}=) ; 7.29-7.15(\mathrm{~m}, 5 \mathrm{arom} . \mathrm{H}) ; 1.02(\mathrm{~s}, t-\mathrm{Bu}) ;-0.09$ ( $\left.s, \mathrm{Me}_{2} \mathrm{Si}\right) .{ }^{13} \mathrm{C}$ NMR: 
158.9 (d, HC=); 142.4 (s, arom. C); 129.6 (d, 3 arom. $C) ; 129.5$ (d, 2 arom. C); 111.0 (s, SiC=); 29.9 (q, Mes C); 0.0 (s, Mes2 Si). Cl-MS: $345\left([M+\mathrm{H}]^{+}\right)$.

2.3. (E)(tert-Butyl)(1-iodo-4-phenylbut-1-enyl)dimethylsilane (8c). Analogeously to $8 \mathbf{a}, 6 \mathbf{c}$ $(2.24 \mathrm{~g}, 9.18 \mathrm{mmol})$, reacted with DIBAH $(11.46 \mathrm{ml})$ and iodine $(3.49 \mathrm{~g}, 13.77 \mathrm{mmol})$, gave after distillation (bulb-to-bulb, $100^{\circ} \mathrm{C} / 10^{-4}$ torr) $8 \mathrm{c}(2.36 \mathrm{~g}, 6.34 \mathrm{mmol}, 69 \%)$ as a slightly yellow oil. IR: $3080 w, 3060 w, 3020 m, 2950 s, 2925 s, 2890 m, 2850 s, 1600 w, 1580 m, 1490 m, 465 m, 1460 m$, $1450 \mathrm{~m}, 1400 \mathrm{w}, 1385 \mathrm{w}, 1360 \mathrm{~m}, 1340 \mathrm{w}, 1260 \mathrm{~s}, 1250 \mathrm{~s}, 1150 \mathrm{w}, 1120 \mathrm{~m}, 1080 \mathrm{w}, 1030 \mathrm{w}, 1005 \mathrm{w}$, $935 w, 905 w, 865 m, 835 s, 820 s, 810 s, 775 m, 765 s, 750 s, 730 m, 695 s, 670 m$. ${ }^{1} \mathrm{H}$ NMR: $7.35(t, j$ $=7.8, \mathrm{HC}=) ; 7.33-7.11(\mathrm{~m}, 10$ arom. $\mathrm{H}) ; 2.65(\mathrm{~m}, \mathrm{PhCH} 2) ; 2.31\left(\mathrm{~m}, \mathrm{CH}_{2} \mathrm{HC}=\right) ; 0.93(\mathrm{~s}, t$-Bu $) ; 0.24$ (s, Me $2 \mathrm{Si}$ ). ${ }^{13} \mathrm{C}$ NMR: 158.5 (d, HC=); 141.9 (s, arom. C); 129.6, 129.4 (2d, each 2 arom. $\left.C\right) ; 127.2$ (d, arom. C); 104.4 (s, $\mathrm{SiC}=) ; 39.1\left(t, \mathrm{PhCH}_{2}\right) ; 36.4\left(t, \mathrm{CH}_{2} \mathrm{HC}=\right) ; 28.3\left(q, \mathrm{Me}_{3} \mathrm{C}\right) ; 19.6\left(\mathrm{~s}, \mathrm{Me}_{3} \mathrm{C}\right) ; 0.0$ $\left(q, \mathrm{Me}_{2} \mathrm{Si}\right)$. Cl-MS: $390\left(\left[\mathrm{M}+\mathrm{NH}_{4}\right]^{+}\right)$.

2.4. (E)-[(Benzyloxy)methyl](tert-butyl)(1-iodoprop-1-enyl)methylsilane (9a). Analogeously to $8 \mathrm{a}, 7 \mathrm{a}(0.88 \mathrm{~g}, 3.38 \mathrm{mmol})$, reacted with DIBAH $(3.60 \mathrm{ml})$ and iodine $(1.12 \mathrm{~g}, 4.40 \mathrm{mmol})$, gave after chromatography (hexane/ $\left.\mathrm{CH}_{2} \mathrm{Cl}_{2} 1: 1\right) 9 a(0.95 \mathrm{~g}, 2.45 \mathrm{mmol}, 72 \%)$ as a slightly yellow oil. IR $\left(\mathrm{CHCl}_{3}\right): 3080 \mathrm{w}, 3060 \mathrm{w}, 3020 \mathrm{~m}, 2970 \mathrm{~s}, 2920 \mathrm{~s}, 2825 \mathrm{~s}, 2810 \mathrm{~m}, 1580 \mathrm{~m}, 1490 \mathrm{~m}, 1460 \mathrm{~s}, 1375 \mathrm{~m}$, $1360 \mathrm{~m}, 1320 w, 1250 \mathrm{~s}, 1205 \mathrm{~m}, 1090 \mathrm{~s}, 1070 \mathrm{~s}, 1025 \mathrm{~m}, 1005 \mathrm{~m}, 980 w, 935 w, 900 w, 830 s, 785 s$, 732s, 695s. ${ }^{1} \mathrm{H}$ NMR: $7.41(q, J=7.4, \mathrm{HC}=) ; 7.37-7.23(\mathrm{~m}, 5$ arom. $\mathrm{H}) ; 4.50\left(\mathrm{~s}, \mathrm{PhCH}_{2} \mathrm{O}\right) ; 3,46$, $3.40\left(A B, J=13.0, \mathrm{SiCH}_{2} \mathrm{O}\right) ; 1.70(d, J=7.4, \mathrm{MeHC}=) ; 1.02(s, t-\mathrm{Bu}) ; 0.19(s, \mathrm{MeSi})$. ${ }_{3} \mathrm{C}$ NMR: $154.0(d, H C=) ; 138.8(s$, arom. C); $128.4,127.8$ (2d, each 2 arom. C); 127.6 (d, arom. C) $99.6(s$, $\mathrm{SiC}=) ; 77.4\left(t, \mathrm{PhCH}_{2} \mathrm{O}\right) ; 63.0\left(t, \mathrm{SiCH}_{2} \mathrm{O}\right) ; 27.8$ (q, MeHC=); $20.0\left(q, \mathrm{Me}_{3} \mathrm{C}\right) ; 19.1\left(\mathrm{~s}, \mathrm{Me}_{3} \mathrm{C}\right) ;-3.7$ (q, MeSi). Cl-MS: $406\left(\left[\mathrm{M}+\mathrm{NH}_{4}\right]^{+}\right)$.

2.5. (E)-[(Benzyloxy)methyl](tert-butyl)(1-iodo-2-phenylethenyl)methylsilane (9b). Analogeously to $8 \mathrm{a}, 7 \mathrm{~b}(2.28 \mathrm{~g}, 7.09 \mathrm{mmol})$, reacted with DIBAH $(7.10 \mathrm{ml}$ of a $1.5 \mathrm{M}$ solution in toluene) and iodine $\left(3.24 \mathrm{~g}, 12.77 \mathrm{mmol}\right.$ ), gave after chromatography (hexane/ $\left.\mathrm{CH}_{2} \mathrm{Cl}_{2} 1: 1\right) 9 \mathrm{~b}(2.65 \mathrm{~g}, 5.88$ mmol, $83 \%)$ as a slightly yellow oil. IR: $3080 w, 3060 \mathrm{~m}, 3020 \mathrm{~m}, 2950 \mathrm{~s}, 2920 \mathrm{~s}, 2880 \mathrm{~s}, 2850 \mathrm{~s}$, $2810 \mathrm{~m}, 1600 \mathrm{w}, 1565 \mathrm{w}, 1490 \mathrm{~m}, 1470 \mathrm{~s}, 1460 \mathrm{~s}, 1440 \mathrm{~m}, 1405 \mathrm{w}, 1390 \mathrm{w}, 1375 \mathrm{~m}, 1360 \mathrm{~m}, 1300 \mathrm{w}$, $1250 s, 1200 w, 1175 w, 1155 w, 1105 s, 1090 s, 1070 s, 1025 m, 1010 w, 980 w, 885 w, 875 w, 850 w$, $825 \mathrm{~s}, 790 \mathrm{~s}, 775 \mathrm{~m}, 750 \mathrm{~s}, 730 \mathrm{~s}, 695 \mathrm{~s}$. ${ }^{1} \mathrm{H}$ NMR: 8.56 ( $\left.\mathrm{s}, \mathrm{HC}=\right) ; 7.35-7.17$ (m, 10 arom. H); 4.33, $4.27\left(A B, J=12.1, \mathrm{PhCH}_{2} \mathrm{O}\right) ; 3.14,3.01\left(\mathrm{AB}, J=13.0, \mathrm{SiCH}_{2} \mathrm{O}\right) ; 1.08(s, t-\mathrm{Bu}) ; 0.03(s, \mathrm{MeSi}) .{ }^{13} \mathrm{C}$ NMR: $157.8(d, \mathrm{HC}=) ; 140.5$ (s, arom. $C$ ); 138.8 (s, arom. C); 128.1 (d, 2 arom. $C) ; 127.8$ (d, 3 arom. C); $127.7,127.5$ (2d, each 2 arom. C); 127.2 (d, arom. C); 105.6 (s, SiC=); $76.8\left(t, \mathrm{PhCH}_{2} \mathrm{O}\right.$ ); 62.2 $\left(t, \mathrm{SiCH}_{2} \mathrm{O}\right) ; 28.4$ (q, Mes $\left.\mathrm{C}\right) ; 18.2\left(s, \mathrm{Me}_{3} C\right) ;-4.5$ (q, MeSi). Cl-MS: $468\left(10,\left[\mathrm{M}^{-\mathrm{NH}_{4}}\right]^{+}\right) ; 238(100)$.

3. Preparation of Silylated Allylic Alcohols. - 3.1. (Z)-2-[(tert-Butyl)dimethy/sily/]-1phenylbut-2-en-1-ol (10a). To a solution of $8 \mathrm{a}(2.00 \mathrm{~g}, 7.09 \mathrm{mmol})$ in $\mathrm{Et}_{2} \mathrm{O}(15 \mathrm{ml})$ was slowly added BuLi $\left(7.8 \mathrm{ml}, 2 \mathrm{~N}\right.$ in pentane) at $-80^{\circ} \mathrm{C}$. It was stirred for $1 \mathrm{~h}$, and benzaldehyde $(2.00 \mathrm{ml}$, $18.70 \mathrm{mmol}$, dissolved in $1 \mathrm{ml}$ of $\mathrm{Et}_{2} \mathrm{O}$ ) was added dropwise. The temperature was slowly raised to $-50^{\circ} \mathrm{C}(15 \mathrm{~min})$ and a saturated aqueous $\mathrm{NH}_{4} \mathrm{Cl}$ solution $(10 \mathrm{ml})$ was added. The aqueous layer was extracted with $\mathrm{Et}_{2} \mathrm{O}$, and the combined organic phases were dried over $\mathrm{MgSO}_{4}$ and evaporated. The crude product gave after chromatography (hexane/ethyl acetate $25: 1) 10 \mathrm{a}(1.50 \mathrm{~g}, 57.47$ $\mathrm{mmol}, 81 \%)$ as a colorless oil. IR $\left(\mathrm{CHCl}_{3}\right) ; 3590 \mathrm{~m}, 3420 \mathrm{br}, 3080 \mathrm{w}, 3060 \mathrm{w}, 2990 \mathrm{w}, 2950 \mathrm{~s}, 2920 \mathrm{~s}$, $2880 s, 2850 s, 2800 w, 2770 w, 2700 w, 1950 w, 1645 w, 1610 m, 1600 m, 1575 w, 1490 w, 1460 m$, $1445 \mathrm{~m}, 1400 w, 1385 w, 1375 w, 1360 \mathrm{~m}, 1205 w, 1250 \mathrm{~s}, 1200 \mathrm{~s}, 1165 w, 1130 \mathrm{~m}, 1090 \mathrm{~m}, 1030 \mathrm{~m}$, $1005 s, 935 w, 925 w, 855 m, 830 s, 820 s$. ' $\mathrm{H}$ NMR: $7.44-7.24$ ( $m, 5$ arom. H); 6.47 ( $q, \mathrm{HC}=) ; 5.34(\mathrm{~s}$, $\mathrm{CH}(\mathrm{OH})) ; 1.87(d, J=6.8, M e H C=) ; 0.91(s, t-\mathrm{Bu}) ; 0.12,0.05$ (2s, Me2si). ${ }^{13} \mathrm{C}$ NMR: 143.7 (s, arom. C); 140.61 (d, HC=); 140.55 (s, SiC=); 128.0 (d, 2 arom. C); 127.0 (d, 3 arom. C); 77.4 (d, $\mathrm{CH}(\mathrm{OH})$ ); $27.2\left(q, \mathrm{Me}_{3} \mathrm{C}\right) ; 18.6(q, \mathrm{MeHC}=) ; 18.5\left(s, \mathrm{Me}_{3} \mathrm{C}\right) ;-3.2 ;-3.4$ (2q, Mes Si). Cl-MS: $245([\mathrm{M}+\mathrm{H}-$ $\left.\left.\mathrm{H}_{2} \mathrm{O}\right]^{+}\right)$.

3.2. (E)-2-[(tert-Butyl)dimethylsilyl]-1-phenylbut-2-en-1-ol (10b). Analogeously to 10a, 8a $(3.34 \mathrm{~g}, 11.84 \mathrm{mmol})$, reacted with BuLi $(13.0 \mathrm{ml})$ in THF at $-5^{\circ} \mathrm{C}$ for $2 \mathrm{~h}$ and with benzaldehyde at $-5^{\circ} \mathrm{C}$ for $10 \mathrm{~min}$, gave after chromatography (hexane/ethyl acetate $\left.25: 1\right) 10 \mathrm{~b}(2.30 \mathrm{~g}, 8.81 \mathrm{mmol}$, $74 \%)$ as a colorless oil. ${ }^{1} \mathrm{H}$ NMR: $7.50-7.17(\mathrm{~m}, 5$ arom. $\mathrm{H}) ; 6.14(q, \mathrm{HC}=) ; 5.77(\mathrm{~s}, \mathrm{CH}(\mathrm{OH})) ; 1.76(d$, $J=6.8, M e H C=) ; 0.88(s, t-B u) ; 0.04,-0.08\left(2 s, \mathrm{Me}_{2} \mathrm{Si}\right)$.

3.3. (Z)-2-[(tert-Butyl)dimethylsilyl]-1-phenyipent-1-en-3-ol (10c) and (E)-2-[(tert-Butyl)dimethylsilyl]-1-phenylpent-1-en-3-ol (10d). Analogeously to $10 \mathrm{a}, 8 \mathrm{~b}(1.00 \mathrm{~g}, 2.91 \mathrm{mmol})$, reacted with BuLi $(2.90 \mathrm{ml})$ in $\mathrm{Et}_{2} \mathrm{O}$ at $-80^{\circ} \mathrm{C}$ for $1 \mathrm{~h}$ and with propionaldehyde $(0.43 \mathrm{ml}, 5.81 \mathrm{mmol})$ at $-80^{\circ} \mathrm{C}$ for 
$10 \mathrm{~min}$, gave after chromatography (hexane/ethyl acetate 25:1) 10d (64 mg, first eluting) and 10c (632 mg, second eluting) as colorless oils (overall $2.52 \mathrm{mmol}, 87 \%$ ).

Data of 10c: IR: $3600 \mathrm{~m}, 3450 \mathrm{br}, 3080 \mathrm{w}, 3050 \mathrm{w}, 2950 \mathrm{~s}, 2930 \mathrm{~s}, 2890 \mathrm{~s}, 2880 \mathrm{~s}, 2850 \mathrm{~s}$, $1590 m, 1485 w, 1460 m, 1440 w, 1400 w, 1485 w, 1475 w, 1460 m, 1285 w, 1250 m, 1230 m, 1115 m$, $1085 m, 1065 m, 1005 m, 980 w, 955 m, 935 w, 925 w, 885 w, 860 m, 835 s, 815 s .{ }^{1} \mathrm{H}$ NMR: $7.78(s$, $\mathrm{HC}=) ; 7.38-2.23(\mathrm{~m}, 5$ arom. $\mathrm{H}) ; 4.38(d d, J=8.4,3.6, \mathrm{CH}(\mathrm{OH})) ; 1.85-1.59\left(\mathrm{~m}, \mathrm{MeCH}_{2}\right) ; 1.13(t, J=$ 7.4, $\mathrm{MeCH}_{2}$ ); 0.98 ( $\left.s, t-\mathrm{Bu}\right) ;-0.04,-0.17$ (2s, Mes $\mathrm{Si}$ ). ${ }^{13} \mathrm{C} \mathrm{NMR}: 146.3$ (s, arom. C); 142.5 (d, HC=); 140.1 (s, SiC=); 128.4, 127.4 (2d, each 2 arom. C); 126.7 (d, arom. C); $74.9(d, \mathrm{CH}(\mathrm{OH})) ; 31.6(t$, $\left.\mathrm{MeCH}_{2}\right) ; 27.6$ (q, Mes $\left.\mathrm{C}\right) ; 18.0\left(\mathrm{~s}, \mathrm{Me}_{3} \mathrm{C}\right) ; 11.0$ (q, MeCH 2$) ;-3.5,-3.7$ (2q, Me $\left.2 \mathrm{Si}\right)$. Cl-MS: 259 $\left(\left[\mathrm{M}+\mathrm{H}-\mathrm{H}_{2} \mathrm{O}\right]^{+}\right)$.

Data of 10d: IR: 3600 br., $3480 \mathrm{br} ., 3080 w, 3050 w, 3020 w, 2960 s, 2930 s, 2890 s, 2880 s$, $2850 \mathrm{~s}, 2800 \mathrm{w}, 1590 \mathrm{w}, 1570 \mathrm{w}, 1490 \mathrm{~m}, 1470 \mathrm{~m}, 1460 \mathrm{~m}, 1440 \mathrm{w}, 1410 \mathrm{w}, 1390 \mathrm{w}, 1350 \mathrm{w}, 1290 \mathrm{w}$, $1250 \mathrm{~s}, 1110 \mathrm{~m}, 1065 \mathrm{~m}, 1030 \mathrm{~m}, 1010 \mathrm{~m}, 965 \mathrm{~m}, 935 \mathrm{w}, 920 \mathrm{w}, 900 \mathrm{w}, 835 \mathrm{~s}, 820 \mathrm{~s}, 810 \mathrm{~s}, 770 \mathrm{~s}$, $750 \mathrm{~m}$, 700s. ${ }^{1} \mathrm{H}$ NMR: $7.38-7.24(\mathrm{~m}, 5$ arom. $\mathrm{H}) ; 6.90(\mathrm{~s}, \mathrm{HC}=) ; 4.68(d d, J=8.3,3.2, \mathrm{CH}(\mathrm{OH})$ ); $1.69\left(m, \mathrm{MeCH}_{2}\right) ; 1.02\left(s, t\right.$-Bu); $0.99\left(t, J=7.6, \mathrm{MeCH}_{2}\right) ; 0.30,0.25\left(2 s, \mathrm{Me}_{2} \mathrm{Si}\right) .{ }^{13} \mathrm{C}$ NMR: 146.5 (s, arom. C); 140.4 (d, HC=) $138.2(s, \mathrm{SiC}=) ; 128.6,128.0$ (2d, each 2 arom C); 126.8 (d, arom. C); $73.9(d, \mathrm{CH}(\mathrm{OH})) ; 30.6\left(t, \mathrm{MeCH}_{2}\right) ; 27.4\left(q, \mathrm{Me}_{3} \mathrm{C}\right) ; 17.4\left(s, \mathrm{Me}_{3} \mathrm{C}\right) ; 10.6\left(q, \mathrm{MeCH}_{2}\right) ;-3.5$ (q, $\left.\mathrm{Me}_{2} \mathrm{Si}\right)$. Cl-MS: $259\left(100\left[\mathrm{M}+\mathrm{H}-\mathrm{H}_{2} \mathrm{O}\right]^{+}\right)$.

3.4. (Z)-2-[(tert-Butyl)dimethylsilyl]-1,3-diphenylprop-2-en-1-ol (10e). Analogeously to 10a, $8 \mathrm{~b}(1.00 \mathrm{~g}, 2.91 \mathrm{mmol})$, reacted with BuLi $(7.40 \mathrm{ml})$ in $\mathrm{Et}_{2} \mathrm{O}$ at $-80^{\circ} \mathrm{C}$ for $1 \mathrm{~h}$ and with benzaldehyde $(1.58 \mathrm{ml}, 14.83 \mathrm{mmol})$ at $-80^{\circ} \mathrm{C}$ for $10 \mathrm{~min}$, gave after chromatography (hexane/ethyl acetate $25: 1$ ) $10 \mathrm{e}(1.80 \mathrm{~g}, 5.55 \mathrm{mmol}, 82 \%)$ as a colorless oil. IR: $3750 \mathrm{br}$., $3480 \mathrm{br}$., $3080 \mathrm{w}, 3050 \mathrm{~m}, 3020 \mathrm{~m}$, $2950 \mathrm{~s}, 2920 \mathrm{~s}, 2880 \mathrm{~s}, 2850 \mathrm{~s}, 2150 \mathrm{w}, 1695 \mathrm{~m}, 1595 \mathrm{~m}, 1580 \mathrm{~m}, 1570 \mathrm{w}, 1485 \mathrm{~m}, 1465 \mathrm{~m}, 1445 \mathrm{~m}$, $1405 w, 1385 m, 1360 m, 1310 w, 1250 s, 1200 w, 1170 w, 1085 w, 1065 m, 1445 m, 1405 w, 1385 m$, $1360 \mathrm{~m}, 1310 w, 1250 \mathrm{~s}, 1200 \mathrm{w}, 1170 \mathrm{w}, 1085 \mathrm{w}, 1065 \mathrm{~m}, 1025 \mathrm{~s}, 1010 \mathrm{~s}, 935 \mathrm{w}, 920 \mathrm{w}, 880 \mathrm{w}, 830 \mathrm{~s}$, $820 s, 810 s, 770 s, 755 s, 745 s, 730 w, 700 s .{ }^{1} \mathrm{H}$ NMR: $7.67(\mathrm{~s}, \mathrm{HC}=) ; 7.49-7.19(\mathrm{~m}, 10$ arom. $\mathrm{H})$; $5.58(s, \mathrm{CH}(\mathrm{OH})) ; 0.87(s, t-\mathrm{Bu}) ;-0.20,-0.25\left(2 s, \mathrm{Me}_{2} \mathrm{Si}\right) . \mathrm{Cl}-\mathrm{MS}: 307\left(\left[\mathrm{M}+\mathrm{H}-\mathrm{H}_{2} \mathrm{O}\right]^{+}\right)$.

3.5. (E)-2-[(tert-Butyl)dimethylsilyl]-1,3-diphenylprop-2-en-1-ol (10f). Analogeously to 10a, $8 \mathrm{~b}(3.30 \mathrm{~g}, 9.71 \mathrm{mmol})$, reacted with BuLi $(10.7 \mathrm{ml})$ in THF at $-5^{\circ} \mathrm{C}$ for $2 \mathrm{~h}$ and with benzaldehyde $\left(2.26 \mathrm{mi}, 21.34 \mathrm{mmol}\right.$ ) at $-5^{\circ} \mathrm{C}$ for $10 \mathrm{~min}$, gave after chromatography (hexane/ethyl acetate 25:1) $10 \mathrm{f}(2.50 \mathrm{~g}, 7.76 \mathrm{mmol}, 79 \%)$ as a colorless oil. IR $\left(\mathrm{CHCl}_{3}\right): 3600 \mathrm{~m}, 3450 \mathrm{br}$., $3080 \mathrm{w}, 3060 \mathrm{~m}$, $3020 \mathrm{w}, 3000 \mathrm{~m}, 2950 \mathrm{~s}, 2930 \mathrm{~s}, 2890 \mathrm{~s}, 2850 \mathrm{~s}, 1500 \mathrm{~s}, 1650 \mathrm{w}, 1595 \mathrm{~m}, 1570 \mathrm{w}, 1490 \mathrm{~s}, 1470 \mathrm{~m}$, $1460 \mathrm{~m}, 1450 \mathrm{~m}, 1410 \mathrm{w}, 1390 \mathrm{w}, 1360 \mathrm{~m}, 1310 \mathrm{w}, 1250 \mathrm{~s}, 1215 \mathrm{w}, 1170 \mathrm{~m}, 1095 \mathrm{w}, 1065 \mathrm{~m}, 1015 \mathrm{~s}$, $920 w, 885 w, 825 s, 700 s .{ }^{1} \mathrm{H}$ NMR: $7.45-7.20(\mathrm{~m}, 10 \mathrm{arom} . \mathrm{H}) ; 7.14(\mathrm{~s}, \mathrm{HC}=) ; 5.94(\mathrm{~s}, \mathrm{CH}(\mathrm{OH})$ ); 0.96 ( $s, t$-Bu); 0.13, -0.19 (2s, Me $2 \mathrm{Si}) .{ }^{13} \mathrm{C}$ NMR: 143.9 ( $s$, arom. C); 142.8 (s, arom. C); $141.4(d$, $\mathrm{HC}=$ ); 137.0 (s, SiC=); 127.9, 127.7 (2d, each. 2 arom. C); 127.6 (d, arom. C), 126.8, 126.4 (2d, each 2 arom. C); 125.7 (d, arom. C); $71.6(d, \mathrm{CH}(\mathrm{OH})) ; 26.9\left(q, \mathrm{Me}_{3} \mathrm{C}\right) ; 17.0\left(\mathrm{~s}, \mathrm{Me}_{3} \mathrm{C}\right) ;-3.8,-5.0$ (2q, $\left.\mathrm{Me}_{2} \mathrm{Si}\right) . \mathrm{Cl}-\mathrm{MS}: 307\left(\left[\mathrm{M}+\mathrm{H}-\mathrm{H}_{2} \mathrm{O}\right]^{+}\right)$.

3.6. (Z)-2-((tert-Butyl)dimethylsilyl]-4-methyl-1-phenylpent-1-en-3-ol (10g). Analogeously to $10 \mathrm{a}, 8 \mathrm{~b}(1.00 \mathrm{~g}, 3.16 \mathrm{mmol})$, reacted with BuLi $(3.16 \mathrm{ml})$ in $\mathrm{Et}_{2} \mathrm{O}$ at $-80^{\circ} \mathrm{C}$ for $1 \mathrm{~h}$ and with isobutyraldehyde $\left(455 \mathrm{mg}, 6.32 \mathrm{mmol}\right.$ ) at $-80^{\circ} \mathrm{C}$ for $10 \mathrm{~min}$, gave after chromatography (hexane/ethyl acetate $25: 1) 10 \mathrm{~g}$ (721 mg, $2.49 \mathrm{mmol}, 82 \%)$ as a colorless oil. ${ }^{1} \mathrm{H}$ NMR: $7.34-7.20$ ( $\mathrm{m}, 5$ arom. $\mathrm{H}$ ); $6.96(s, \mathrm{HC}=) ; 4.36(d, J=9.1, \mathrm{CH}(\mathrm{OH})) ; 1.87\left(\mathrm{~m}, \mathrm{Me}_{2} \mathrm{CH}\right) ; 0.99(s, t-\mathrm{Bu}) ; 0.94,0.73(2 d, j=6.5$, 6.9, $\left.\mathrm{Me}_{2} \mathrm{CH}\right) ; 0.25,0.21\left(2 \mathrm{~s}, \mathrm{Me}_{2} \mathrm{Si}\right)$. Cl-MS: $273\left(\left[\mathrm{M}+\mathrm{H}-\mathrm{H}_{2} \mathrm{O}\right]^{+}\right)$.

3.7. (Z)-2-[(tert-Butyl)dimethylsilyl]-1,5-diphenylpent-2-en-1-ol (10h) and (E)-2-[(tert-Butyl)dimethylsilyl]-1,5-diphenylpent-2-en-1-ol (10i). Analogeously to $10 \mathrm{a}, 8 \mathrm{c}(2.00 \mathrm{~g}, 5.38 \mathrm{mmol})$, reacted with BuLi $(4.0 \mathrm{ml})$ in $\mathrm{Et}_{2} \mathrm{O}$ at $-80^{\circ} \mathrm{C}$ for $1 \mathrm{~h}$ and with benzaldehyde $(0.96 \mathrm{ml}, 9.00 \mathrm{mmol})$ at $-80^{\circ} \mathrm{C}$ for $10 \mathrm{~min}$, gave after chromatography (hexane/ethyl acetate $\left.25: 1\right) 10 \mathrm{i}(100 \mathrm{mg}$, first eluting) and $10 \mathrm{~h}(1.46 \mathrm{~g}$, second eluting) as colorless oils (overall $4.44 \mathrm{mmol}, 83 \%$ ).

Data of 10h: IR: $3550 \mathrm{br} ., 3430 \mathrm{br} ., 3880 \mathrm{w}, 3060 \mathrm{w}, 3020 \mathrm{~m}, 2950 \mathrm{~s}, 2920 \mathrm{~s}, 2890 \mathrm{~s}, 2850 \mathrm{~s}$, $1600 \mathrm{~m}, 1490 \mathrm{~m}, 1470 \mathrm{~m}, 1450 \mathrm{~m}, 1410 \mathrm{w}, 1385 \mathrm{w}, 1360 \mathrm{w}, 1250 \mathrm{~m}, 1150 \mathrm{w}, 1110 \mathrm{w}, 1060 \mathrm{w}, 1030 \mathrm{w}$, $1005 \mathrm{~m}, 940 \mathrm{w}, 915 \mathrm{w}, 890 \mathrm{w}, 835 \mathrm{~s}, 820 \mathrm{~s}, 810 \mathrm{~m}, 775 \mathrm{~m}, 760 \mathrm{~m}, 745 \mathrm{~m}, 700 \mathrm{~s}, 675 \mathrm{~m}$. ${ }^{1} \mathrm{H}$ NMR: $7.41-$ $7.23(m, 10$ arom. $\mathrm{H}) ; 6.55(t, J=7.8, \mathrm{HC}=) ; 5.40(\mathrm{~s}, \mathrm{CH}(\mathrm{OH})) ; 2.82-2.78\left(m, \mathrm{PhCH}_{2}\right) ; 2.68-2.59$ $\left(m, \mathrm{CH}_{2} \mathrm{C}=\right) ; 0.97(s, t-\mathrm{Bu}) ; 0.16,0.07\left(2 \mathrm{~s}, \mathrm{Me}_{2} \mathrm{Si}\right) .{ }^{13} \mathrm{C} \mathrm{NMR:} 145.1$ (d, HC=); 143.5 (s, arom. C); 141.5 (s, arom. C); 140.1 (s, SiC=); 128.4, 128.3 (2d, each 2 arom. C); 128.1 (d, arom. C); 127.2 (d, 
2 arom. C); $125.8\left(d, 3\right.$ arom. C); $65.8(d, \mathrm{CH}(\mathrm{OH})) ; 36.2\left(t, \mathrm{PhCH}_{2}\right) ; 34.7\left(t, \mathrm{CH}_{2} \mathrm{C}=\right) ; 27.0(q$, $\left.\mathrm{Me}_{3} \mathrm{C}\right) ; 18.3\left(\mathrm{~s}, \mathrm{Me}_{3} \mathrm{C}\right) ;-3.2,-3.1(2 q, \mathrm{Me} 2 \mathrm{Si}) . \mathrm{Cl}-\mathrm{MS}: 335\left(60\left[\mathrm{M}+\mathrm{H}-\mathrm{H}_{2} \mathrm{O}\right]^{+}\right)$.

Data of 10i: IR: $3570 \mathrm{~m}, 3470 \mathrm{br}, 3080 \mathrm{w}, 3060 \mathrm{~m}, 3020 \mathrm{~m}, 2950 \mathrm{~s}, 2920 \mathrm{~s}, 2880 \mathrm{~s}, 2850 \mathrm{~s}$, $2730 w, 1700 s, 1650 w, 1600 m, 1580 w, 1490 m, 1470 m, 1460 m, 1450 s, 1405 w, 1385 m, 1360 m$, $1310 m, 1245 s, 1200 m, 1165 m, 1100 w, 1065 w, 1030 m, 1005 m, 965 w, 940 w, 925 w, 880 w, 820 s$, $810 s, 770 s, 745 s, 725 m, 700 s$. ${ }^{1} \mathrm{H}$ NMR: $7.39-7.16(m, 10$ arom. $\mathrm{H}) ; 6.08(t, J=7.4, \mathrm{HC}=) ; 5.62$ ( $s$, $\mathrm{CH}(\mathrm{OH})$ ); 2.84-2.43 (m, PhCH $\left.\mathrm{CH}_{2}\right) ; 0.90$ (s, Mes $\mathrm{C}$ ); 0.05, -0.09 (2s, Me2Si). 13 C NMR: 143.5 (d, $\mathrm{HC}=$ ); 142.5 ( $\mathrm{s}$, arom. C); $141.4(\mathrm{~s}, \mathrm{SiC}=) ; 134.4(\mathrm{~s}$, arom. $\mathrm{C}) ; 128.6,128.2$ (2d, each 2 arom. $\mathrm{C}$ ); 128.0 (d, arom. C); 126.6 (d, 2 arom. C); 126.0 (d, 3 arom. C); 72.4 (d, $\mathrm{CH}(\mathrm{OH})$ ); $35.4\left(t, \mathrm{PhCH}_{2}\right.$ ); $31.7\left(t, \mathrm{CH}_{2} \mathrm{C}=\right) ; 27.1\left(q, \mathrm{Me}_{3} \mathrm{C}\right) ; 17.2\left(s, \mathrm{Me}_{3} \mathrm{C}\right) ;-4.0,-4.6\left(2 q, \mathrm{Me}_{2} \mathrm{Si}\right)$. Cl-MS: 335 (60 [M+H$\left.\left.\mathrm{H}_{2} \mathrm{O}\right]^{+}\right)$.

3.8. (Z)-2-\{[(Benzyloxy)methyl](tert-butyl)methylsilyl\}-1-phenylbut-2-en-1-ol (11a and 11b). Analogeously to $10 \mathrm{a}, 9 \mathrm{a}(0.93 \mathrm{~g}, 2.40 \mathrm{mmol})$, reacted with BuLi $(2.40 \mathrm{ml})$ in $\mathrm{Et}_{2} \mathrm{O}$ at $-80^{\circ} \mathrm{C}$ for $1 \mathrm{~h}$ and with benzaldehyde $\left(0.51 \mathrm{ml}, 4.79 \mathrm{mmol}\right.$ ) at $-80^{\circ} \mathrm{C}$ for $10 \mathrm{~min}$, gave after chromatography (hexane/ethyl acetate $25: 1) 11 \mathrm{a}$ ( $410 \mathrm{mg}$, first eluting) and $11 \mathrm{~b}(200 \mathrm{mg}$, second eluting) as colorless oils (overall $1.66 \mathrm{mmol}, 69 \%$ ).

Data of 11a: IR $\left(\mathrm{CHCl}_{3}\right): 3080 \mathrm{w}, 3060 \mathrm{w}, 3359 \mathrm{br} ., 3000 \mathrm{~s}, 2920 \mathrm{~s}, 2860 \mathrm{~s}, 1620 \mathrm{~m}, 1465 \mathrm{~s}$, $1550 \mathrm{~s}, 1380 \mathrm{~m}, 1360 \mathrm{~m}, 1255 \mathrm{~s}, 1090 \mathrm{~s}, 1060 \mathrm{~s} 1030 \mathrm{~m}, 1010 \mathrm{~s}$. $940 \mathrm{w}, 905 \mathrm{w}, 865 \mathrm{w}, 825 \mathrm{~s} .{ }^{1} \mathrm{H}$ NMR: 7.41-7.25 (m, 10 arom. H); $6.31(q, J=7.0, \mathrm{HC}=) ; 5.30(d, J=8.2, \mathrm{CH}(\mathrm{OH})) ; 4.72(d, \mathrm{OH}) ; 4.27$, $4.43\left(A B, J=11.7, \mathrm{PhCH}_{2} \mathrm{O}\right) ; 3.25,2.61\left(A B, J=12.7, \mathrm{SiCH}_{2} \mathrm{O}\right) ; 1.88(d, \mathrm{MeHC}=) ; 1.02(s, t-\mathrm{Bu}) ;$ $0.16(\mathrm{~s}, \mathrm{MeSi}) .{ }^{13} \mathrm{C}$ NMR: $144.6(\mathrm{~s}$, arom. $\mathrm{H}) ; 143.0(\mathrm{~d}, \mathrm{HC}=) ; 139.7(\mathrm{~s}, \mathrm{SiC}=) ; 137.2(\mathrm{~s}$, arom. $\mathrm{H}) ;$ 128.3, 127.8 ( $2 d$, each 2 arom. C); 127.7 (d, arom. C); 126.5 (d, arom. C); 126.2 (d, 3 arom. C); 79.6 $(d, \mathrm{CH}(\mathrm{OH})) ; 77.1\left(t, \mathrm{PhCH}_{2} \mathrm{O}\right) ; 61.9\left(t, \mathrm{SiCH}_{2} \mathrm{O}\right) ; 27.8$ (q, Mes $\left.\mathrm{C}\right) ; 18.8\left(s, \mathrm{Me}_{3} \mathrm{C}\right) ; 18.7(q, \mathrm{MeHC}=) ;$ -5.8 (q, MeSi). Cl-MS: $369\left(30,[M+H]^{+}\right), 238(100)$.

Data of 11b: IR $\left(\mathrm{CHCl}_{3}\right): 3350 \mathrm{br} .3080 \mathrm{w}, 3060 \mathrm{w}, 3000 \mathrm{~s}, 2930 \mathrm{~s}, 2860 \mathrm{~s}, 1615 \mathrm{~m}, 1495 \mathrm{~m}$, $1465 \mathrm{~s}, 1450 \mathrm{~s}, 1380 \mathrm{~m}, 1365 \mathrm{~m}, 1255 \mathrm{~s}, 1090 \mathrm{~s}, 1065 \mathrm{~s}, 1030 \mathrm{~m}, 1010 \mathrm{~s}, 940 \mathrm{w}, 905 \mathrm{w}, 865 \mathrm{w}, 825 \mathrm{~s}$, 700s. $1 \mathrm{H}$ NMR: $7.39-7.20(\mathrm{~m}, 10$ arom. $\mathrm{H}) ; 6.33(q, \mathrm{HC}=) ; 5.33(\mathrm{~s}, \mathrm{CH}(\mathrm{OH})) ; 4.51,4.46(A B, J=$ 11.8, $\left.\mathrm{PhCH} \mathrm{H}_{2} \mathrm{O}\right) ; 3.28,3.17\left(\mathrm{AB}, J=12.7, \mathrm{SiCH}_{2} \mathrm{O}\right) ; 1.83(d, J=7.2, \mathrm{MeHC}=) ; 0.83(s, t-\mathrm{Bu}) ; 0.21$ $(s, \mathrm{MeSi}) .{ }^{13} \mathrm{C}$ NMR: 142.7 ( $s$, arom. C); 141.5 (d, HC=); 139.3 ( $\left.s, \mathrm{SiC}=\right) ; 136.6$ ( $s$, arom. C); 127.2, 126.8 (2d, each 2 arom. C); 126.7 (d, arom. C); 126.5, 125.7 (2d, each 2 arom. C); 125.6 (d, arom. C); $78.1(d, \mathrm{CH}(\mathrm{OH})) ; 76.2\left(t, \mathrm{PhCH}_{2} \mathrm{O}\right) ; 61.1\left(t, \mathrm{SiCH}_{2} \mathrm{O}\right) ; 26.7\left(q, \mathrm{Me}_{3} \mathrm{C}\right) ; 17.8(q, \mathrm{MeHC}=) ; 17.3(s$, $\left.\mathrm{Me}_{3} \mathrm{C}\right) ;-6.3(q, \mathrm{MeSi})$. Cl-MS: $369\left(30,[\mathrm{M}+\mathrm{H}]^{+}\right), 238(100)$.

3.9. (E)-2-\{[(Benzyloxy)methyl](tert-butyl)methylsilyl\}-1-phenylbut-2-en-1-ol (11c and 11d). Analogeously to $10 \mathrm{a}, 9 \mathrm{a}(1.40 \mathrm{~g}, 3.67 \mathrm{mmol})$, reacted with BuLi $(4.0 \mathrm{ml})$ in THF at $-5^{\circ} \mathrm{C}$ for $2 \mathrm{~h}$ and with benzaldehyde $(0.90 \mathrm{ml}, 7.94 \mathrm{mmol})$ at $-5^{\circ} \mathrm{C}$ for $10 \mathrm{~min}$, gave after chromatography (hexane/

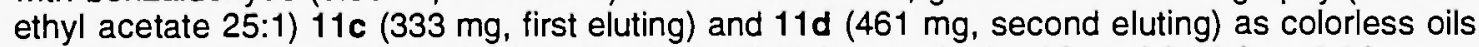
(overall $2.16 \mathrm{mmol}, 60 \%$ ). Optically active $11 \mathrm{c}$ and $11 \mathrm{~d}$ were obtained from (-)-13 (see 6.2.).

Data of $(-)-\left(R_{S i}\right)-11 \mathrm{c}:[\alpha]_{\mathrm{D}}^{23}=-15 \pm 2(\mathrm{c}=1.1 \mathrm{THF})$. IR: $3390 \mathrm{br}, 3080 \mathrm{w}, 3060 \mathrm{~m}, 3020 \mathrm{~m}$, $3000 \mathrm{w}, 2960 \mathrm{~s}, 2920 \mathrm{~s}, 2880 \mathrm{~s}, 2850 \mathrm{~s}, 2810 \mathrm{~m}, 2600 \mathrm{~m}, 1765 \mathrm{w}, 1490 \mathrm{~m}, 1460 \mathrm{~m}, 1445 \mathrm{~m}, 1430 \mathrm{~m}$, $1380 \mathrm{~m}, 1360 \mathrm{~m}, 1250 \mathrm{~m}, 1205 \mathrm{w}, 1185 \mathrm{~m}, 1175 \mathrm{~m}, 1150 \mathrm{~m}, 1085 \mathrm{~m}, 1065 \mathrm{~s}, 1025 \mathrm{~m}, 1010 \mathrm{~s}, 980 \mathrm{w}$, $940 w, 920 w, 900 w, 875 w, 865 w, 825 s, 810 m, 795 m, 745 s, 720 m, 700 s .{ }^{\dagger} \mathrm{H}$ NMR: $7.49-7.23(m$, 10 arom. $\mathrm{H}) ; 6.25(q, J=6.9, \mathrm{HC}=) ; 5.76(s, \mathrm{CH}(\mathrm{OH})) ; 4.89(\mathrm{~s}, \mathrm{OH}) ; 4.29,4.10(A B, J=11.8$, $\left.\mathrm{PhCH}_{2} \mathrm{O}\right) ; 3.24,2.56\left(A B, J=12.7, \mathrm{SiCH}_{2} \mathrm{O}\right) ; 1.87(d, J=6.8, \mathrm{MeHC}) ; 1.08(s, t-\mathrm{Bu}) ; 0.04(s$, MeSi). ${ }^{13} \mathrm{C}$ NMR: $144.5(s$, arom. $C) ; 142.3$ (s, arom. C); $138.1(d, \mathrm{HC}=) ; 137.2(s, \mathrm{SiC}=) ; 128.3$, 128.1 (2d, each 2 arom. C); 127.8 ( $d$, arom. C); 127.7, 126.6 (2d, each 2 arom. C); 126.0 ( $d$, arom C); $77.0\left(t, \mathrm{PhCH}_{2} \mathrm{O}\right) ; 71.4(d, \mathrm{CH}(\mathrm{OH})) ; 61.2\left(t, \mathrm{SiCH}_{2} \mathrm{O}\right) ; 27.6\left(q, \mathrm{Me}_{3} \mathrm{C}\right) ; 17.6\left(\mathrm{~s}, \mathrm{Me}_{3} \mathrm{C}\right) ; 15.3(q$, MeHC $=) ;-6.6(q, \mathrm{MeSi})$. Cl-MS (isobutane): $351\left(10,\left[\mathrm{M}+\mathrm{H}-\mathrm{H}_{2} \mathrm{O}\right]^{+}\right), 221(100)$.

Data of $(-)-\left(R_{S i}\right)-11 \mathrm{~d}:[\alpha]_{D}^{23}=-13 \pm 2(c=0.8$, THF). IR: $3570 \mathrm{w}, 3390 \mathrm{br} ., 3080 \mathrm{w}, 3060 \mathrm{w}$, $3020 \mathrm{~m}, 2960 \mathrm{~s}, 2920 \mathrm{~s}, 2880 \mathrm{~s}, 2850 \mathrm{~s}, 1600 \mathrm{~m}, 1490 \mathrm{~m}, 1470 \mathrm{~m}, 1460 \mathrm{~m}, 1445 \mathrm{~m}, 1380 \mathrm{~m}, 1360 \mathrm{~m}$, $1250 m, 1205 w, 1190 w, 1175 w, 1140 w, 1080 m, 1060 m, 1025 m, 1010 m, 980 w, 935 w, 915 w$, $900 w, 855 w, 825 m, 800 m, 785 m, 765 m, 750 m, 735 m, 700 s .{ }^{1} \mathrm{H}$ NMR: $7.37-7.25(m, 10$ arom. H); $6.18(q, \mathrm{HC}=) ; 5.82(s, \mathrm{CH}(\mathrm{OH})) ; 4.46,4.37\left(\mathrm{AB}, \mathrm{J}=12.8, \mathrm{PhCH} \mathrm{H}_{2} \mathrm{O}\right) ; 4.27(\mathrm{~s}, \mathrm{OH}) ; 3.19(\mathrm{~s}$, $\left.\mathrm{SiCH}_{2} \mathrm{O}\right) ; 1.88(d, J=6.8, \mathrm{MeHC}=) ; 0.69\left(s, \mathrm{Me}_{3} \mathrm{C}\right) ; 0.13$ (s, MeSi). ${ }^{13} \mathrm{C}$ NMR: 144.0 (s, arom. C); 141.8 (s, arom. C); $139.4(d, \mathrm{HC}=) ; 137.5(\mathrm{~s}, \mathrm{SiC}=) ; 128.3,127.9$ (2d, each 2 arom. C); 127.7 (d, arom. C); 126.4, (d, 2 arom. C) 125.8 (d, 3 arom. C); $77.4\left(t, \mathrm{PhCH}_{2} \mathrm{O}\right) ; 71.5(d, \mathrm{CH}(\mathrm{OH})) ; 61.4(t$, 
$\mathrm{SiCH}_{2} \mathrm{O}$ ); $27.3\left(q, \mathrm{Me}_{3} \mathrm{C}\right) ; 17.1$ (s, $\left.\mathrm{Me}_{3} \mathrm{C}\right) ; 15.6$ ( $\left.q, \mathrm{MeHC}=\right) ;-5.8$ ( $\left.q, \mathrm{MeSi}\right) . \mathrm{Cl}-\mathrm{MS}$ (isobutane): 351 $\left(10,\left[\mathrm{M}+\mathrm{H}-\mathrm{H}_{2} \mathrm{O}\right]^{+}\right), 221(100)$.

3.10. (Z)-4-\{[(Benzyloxy)methyl](tert-butyl)methylsilyl\}-2-methylhex-4-en-3-ol (11e and 11f). Analogeously to $10 \mathrm{a}, 9 \mathrm{a}(375 \mathrm{mg}, 0.97 \mathrm{mmol})$, reacted with BuLi $(2.0 \mathrm{ml})$ in $\mathrm{Et}_{2} \mathrm{O}$ at $-80^{\circ} \mathrm{C}$ for $1 \mathrm{~h}$ and with isobutyraldehyde $(1.39 \mathrm{mg}, 1.93 \mathrm{mmol})$ at $-80^{\circ} \mathrm{C}$ for $10 \mathrm{~min}$, gave after chromatography (hexane/ethyl acetate 25:1) 11e (100 mg, first eluting) and $11 \mathrm{f}(200 \mathrm{mg}$, second eluting) as colorless oils (overall $0.90 \mathrm{mmol}, 93 \%$ ).

Data of 11e: IR $\left(\mathrm{CHCl}_{3}\right): 3400 \mathrm{br}, 3090 \mathrm{w}, 3070 \mathrm{w}, 3030 \mathrm{w}, 3000 \mathrm{~s}, 2960 \mathrm{~s}, 2930 \mathrm{~s}, 2860 \mathrm{~s}$, $2820 \mathrm{~m}, 1610 \mathrm{~m}, 1495 \mathrm{w}, 1465 \mathrm{~m}, 1455 \mathrm{~m}, 1435 \mathrm{~m}, 1380 \mathrm{~m}, 1365 \mathrm{~m}, 1310 \mathrm{w}, 1255 \mathrm{~m}, 1215 \mathrm{w}, 1160 \mathrm{w}$, $1125 w, 1085 m, 1070 s, 1030 m, 1010 m, 990 w, 940 w, 905 w, 860 w, 825 m, 805 m, 700 m .1 \mathrm{H}$ NMR: 7.29-7.26 (m, 5 arom. H); $6.34(q, \mathrm{HC}=) ; 4.45\left(\mathrm{~s}, \mathrm{PhCH}_{2} \mathrm{O}\right) ; 3.63(\mathrm{~m}, \mathrm{CH}(\mathrm{OH})) ; 3.39,3.20(A B, J=$ 12.8, $\left.\mathrm{SiCH}_{2} \mathrm{O}\right) ; 1.75(d, J=7.0, \mathrm{MeHC}=) ; 1.70\left(\mathrm{~m}, \mathrm{Me}_{2} \mathrm{CH}\right) ; 0.97(d, J=6.6, \mathrm{MeCH}) ; 0.94(s, t-\mathrm{Bu})$; $0.75(d, J=6.8, \mathrm{MeCH}) ; 0.13(s, \mathrm{MeSi}) .{ }^{13} \mathrm{C}$ NMR: $140.3(d, \mathrm{HC}=) ; 139.3(s, \mathrm{SiC}=) ; 137.5$ (s, arom. C); 128.2, 127.9 (2d, each 2 arom. C); 127.7 (d, arom. C); 84.1 (d, $\mathrm{CH}(\mathrm{OH})$ ); $77.3\left(t, \mathrm{PhCH}_{2} \mathrm{O}\right.$ ); 62.2 $\left(t, \mathrm{SiCH}_{2} \mathrm{O}\right) ; 33.1\left(d, \mathrm{Me}_{2} \mathrm{CH}\right) ; 27.8\left(q, \mathrm{Me}_{3} \mathrm{C}\right) ; 20.6(q, \mathrm{MeHC}=) ; 19.4(q, \mathrm{MeCH}) ; 18.7$ ( $q$ and $s$, $\left.\mathrm{MeCH}, \mathrm{Me}_{3} \mathrm{C}\right) ;-5.6(q, \mathrm{MeSi}) . \mathrm{Cl}-\mathrm{MS}: 317\left(70,\left[\mathrm{M}+\mathrm{H}-\mathrm{H}_{2} \mathrm{O}\right]^{+}\right), 238(100)$.

Data of 11f: IR $\left(\mathrm{CHCl}_{3}\right)$ : $3500 \mathrm{br}, 3080 \mathrm{w}, 3060 \mathrm{w}, 3010 \mathrm{~m}, 3000 \mathrm{~s}, 2970 \mathrm{~s}, 2920 \mathrm{~s}, 2850 \mathrm{~s}$, $2810 \mathrm{~m}, 1610 \mathrm{~m}, 1490 \mathrm{w}, 1460 \mathrm{~m}, 1450 \mathrm{~m}, 1430 \mathrm{~m}, 1380 \mathrm{~m}, 1360 \mathrm{~m}, 1320 \mathrm{w}, 1310 \mathrm{w}, 1250 \mathrm{~m}, 1210 \mathrm{w}$, $1180 w, 1160 w, 1120 w, 1080 w, 1030 m, 1000 \mathrm{~m}, 980 \mathrm{w}, 930 \mathrm{w}, 880 \mathrm{w}, 860 \mathrm{w}, 820 \mathrm{~s}, 800 \mathrm{~m}, 700 \mathrm{~m}$. ${ }_{1} \mathrm{H}$ NMR: $7.39-7.25(m, 5$ arom. $\mathrm{H}) ; 6.44(q, \mathrm{HC}=) ; 4.48\left(s, \mathrm{PhCH}_{2} \mathrm{O}\right) ; 3.76(d, J=6.9, \mathrm{CH}(\mathrm{OH}))$; 3.53, $3.34\left(A B, J=12.0, \mathrm{SiCH}_{2} \mathrm{O}\right) ; 1.83(d, J=7.0, \mathrm{MeHC}=) ; 1.66\left(\mathrm{~m}, \mathrm{Me}_{2} \mathrm{CH}\right) ; 0.97(s, t-\mathrm{Bu}) ; 0.94$, $0.84\left(2 d, J=6.6,6.8, M e_{2} \mathrm{CH}\right) ; 0.24$ (s, MeSi). ${ }^{13} \mathrm{C}$ NMR: 140.8 (d, HC=); 139.5 (s, SiC=); $138.1(s$, arom. C); 128.1, 127.7 (2d, each 2 arom. C); 127.4 (d, arom. C); $83.2(d, \mathrm{CH}(\mathrm{OH})$ ); $77.3(t$, $\left.\mathrm{PhCH}_{2} \mathrm{O}\right) ; 62.4\left(t, \mathrm{SiCH}_{2} \mathrm{O}\right) ; 33.8\left(d, \mathrm{Me}_{2} \mathrm{CH}\right) ; 27.9\left(q, \mathrm{Me}_{3} \mathrm{C}\right) ; 20.6(q, \mathrm{MeHC}=) ; 18.8(q, \mathrm{MeCH}) ;$ 18.7 (s, Mes $\mathrm{C}) ; 17.6(q, \mathrm{MeCH}) ;-6.2(q, \mathrm{MeSi})$. Cl-MS: $317\left(70,\left[\mathrm{M}+\mathrm{H}-\mathrm{H}_{2} \mathrm{O}\right]^{+}\right), 238(100)$.

3.11. (E)-2-\{[(Benzyloxy)methyl](tert-butyl)methylsilyl\}-1,3-diphenylprop-2-en-1-ol (11 g and $11 \mathrm{~h})$. Analogeously to $10 \mathrm{a}, 9 \mathrm{~b}(1.30 \mathrm{~g}, 2.89 \mathrm{mmol})$, reacted with BuLi $(3.00 \mathrm{ml})$ in $\mathrm{Et}_{2} \mathrm{O}$ at $-80^{\circ} \mathrm{C}$ for $1 \mathrm{~h}$ and with benzaldehyde $(0.70 \mathrm{ml}, 6.70 \mathrm{mmol})$ at $-80^{\circ} \mathrm{C}$ for $10 \mathrm{~min}$, gave after chromatography (hexane/ethyl acetate $25: 1$ ) $11 \mathrm{~g}$ (435 mg, first eluting) and $11 \mathrm{~h}$ (565 $\mathrm{mg}$, second eluting) as colorless oils (overall $2.36 \mathrm{mmol}, 80 \%$ ).

Data of 11g: IR: $3380 \mathrm{br} ., 3080 \mathrm{w}, 3060 \mathrm{~m}, 3020 \mathrm{~m}, 2950 \mathrm{~s}, 2920 \mathrm{~s}, 2880 \mathrm{~s}, 2850 \mathrm{~s}, 2820 \mathrm{~m}$, $1600 \mathrm{w}, 1590 \mathrm{w}, 1570 \mathrm{w}, 1490 \mathrm{~m}, 1470 \mathrm{~m}, 1460 \mathrm{~m}, 1445 \mathrm{~m}, 1430 \mathrm{~m}, 1390 \mathrm{~m}, 1380 \mathrm{~m}, 1360 \mathrm{~m}, 1315 \mathrm{w}$, $1250 \mathrm{~m}, 1220 w, 1200 w, 1185 w, 1175 w, 1155 w, 1085 \mathrm{~m}, 1065 \mathrm{~s}, 1035 \mathrm{~s}, 1025 \mathrm{~s}, 980 w, 925 \mathrm{~m}$, $915 \mathrm{~m}, 905 \mathrm{~m}, 885 w, 825 \mathrm{~s}, 810 \mathrm{~s}, 795 \mathrm{~s}, 755 \mathrm{~s}, 745 \mathrm{~s}, 735 \mathrm{~s}, 720 \mathrm{~m}, 700 \mathrm{~s}$. ${ }^{1} \mathrm{H}$ NMR: $7.50-7.14(\mathrm{~m}, 15$ arom. $\mathrm{H}) ; 7.13(s, \mathrm{HC}=) ; 5.73(d, J=13.5, \mathrm{CH}(\mathrm{OH})) ; 4.91(d, \mathrm{OH}) ; 4.12,3.90(A B, J=11.7$, $\left.\mathrm{PhCH}_{2} \mathrm{O}\right) ; 3.15,2.41\left(A B, J=12.8, \mathrm{SiCH}_{2} \mathrm{O}\right) ; 1.08(s, t-\mathrm{Bu}) ; 0.07(s, \mathrm{MeSi}) .{ }^{13} \mathrm{C}$ NMR: $144.6(s$, arom. C); 143.6 (s, arom. C); $141.5(d, \mathrm{HC}=) ; 137.3(s, \mathrm{SiC}=) ; 137.0$ (s, arom. $\mathrm{C}) ; 128.4,128.3(2 d$, each 2 arom. C); 128.2 (d, arom. C); $128.1,127.8$ (2d, each 2 arom. C); 127.7 (d, arom. C); 127.2, 126.8 (2d, each 2 arom. C); 126.3 (d, arom. C); $77.0\left(t, \mathrm{PhCH}_{2} \mathrm{O}\right) ; 71.6(d, \mathrm{CH}(\mathrm{OH})) ; 60.8(t$, $\left.\mathrm{SiCH}_{2} \mathrm{O}\right) ; 27.6$ (q, Mes $\left.\mathrm{C}\right) ;-6.5$ (q, MeSi). Cl-MS: $413\left(10,\left[\mathrm{M}+\mathrm{H}-\mathrm{H}_{2} \mathrm{O}\right]^{+}\right), 238(100)$.

Data of 11h: IR: $3580 w, 3400 \mathrm{br} ., 3080 \mathrm{w}, 3060 \mathrm{~m}, 3020 \mathrm{~m}, 2960 \mathrm{~s}, 2920 \mathrm{~s}, 2880 \mathrm{~s}, 2850 \mathrm{~s}$, $1600 w, 1590 w, 1570 w, 1490 s, 1470 s, 1460 s, 1450 s, 1390 m, 1370 m, 1360 \mathrm{~m}, 1250 \mathrm{~s}, 1200 w$, $1175 w, 1155 w, 1090 s, 1065 s, 1025 s, 980 w, 925 m, 905 w, 885 w, 820 s, 790 s, 770 s, 760 s, 745 s$, 700s. ${ }^{1} \mathrm{H}$ NMR: $7.37-7.23$ (m, 15 arom. $\left.\mathrm{H}\right) ; 7.21$ (s, $\left.\mathrm{HC}=\right) ; 5,91(\mathrm{~s}, \mathrm{CH}(\mathrm{OH})) ; 4.48,4.38(A B, J=$ 11.7, $\left.\mathrm{PhCH}_{2} \mathrm{O}\right) ; 4.33(s, \mathrm{OH}) ; 3.22,3.12\left(A B, J=12.6, \mathrm{SiCH}_{2} \mathrm{O}\right) ; 0.82(s, t-\mathrm{Bu}) ; 0.18(s, \mathrm{MeSi}) .{ }^{13} \mathrm{C}$ NMR: 143.9 (s, arom. C); 142.9 (d, HC= and probably $s$, arom $C$ ); 137.5 (s, arom. $C$ ); $137.4(s, \mathrm{SiC}=)$; 128.5, 128.3 (2d, each 2 arom. C); 128.2 (d, arom. C); $128.0,127.9$ (2d, each 2 arom. C); $127.7(d$, arom. C); 127.2, 126.2 (2d, each 2 arom. C); 126.0 (d, arom. C); $77.3\left(t, \mathrm{PhCH}_{2} \mathrm{O}\right) ; 71.8$ (d, $\mathrm{CH}(\mathrm{OH})) ; 61.1\left(t, \mathrm{SiCH}_{2} \mathrm{O}\right) ; 27.6\left(q, \mathrm{Me}_{3} \mathrm{C}\right) ;-5.4(q, \mathrm{MeSi}) . \mathrm{Cl}-\mathrm{MS}: 413\left(10,\left[\mathrm{M}+\mathrm{H}-\mathrm{H}_{2} \mathrm{O}\right]^{+}\right), 238$ $(100)$.

4. O-Acetylations. - 4.1. (Z)-2-[(tert-Butyl)dimethylsilyl]-1-phenylbut-2-enyl trifloroacetate (15a): To a solution of $10 \mathrm{a}(200 \mathrm{mg}, 0.76 \mathrm{mmol})$ in pyridine $(5 \mathrm{ml})$ was added trifluoroacetic anhydride $(160 \mathrm{mg}, 1.15 \mathrm{mmol})$. It was stirred for $2 \mathrm{~h}$ at $23^{\circ} \mathrm{C}$ and poured on $\mathrm{H}_{2} \mathrm{O}(10 \mathrm{ml})$. The aqueous layer was extracted with $\mathrm{Et}_{2} \mathrm{O}$, and the combined organic phases were dried over $\mathrm{MgSO}_{4}$ and evaporated to give $15 \mathrm{a}(182 \mathrm{mg}, 0.48 \mathrm{mmol}, 95 \%)$. Further purification proved impossible due to the high instability of the product. ${ }^{1} \mathrm{H}$ NMR: $7.43-7.23(\mathrm{~m}, 5$ arom. $\mathrm{H}) ; 7.06\left(\mathrm{~s}, \mathrm{CF}_{3} \mathrm{CO}_{2} \mathrm{CH}\right) ; 6.20(q$, 
$\mathrm{HC}=) ; 1.57(d, J=6.3, \mathrm{MeHC}=) ; 1.03(s, t$-Bu); 0.33, 0.31 (2s, Me $2 \mathrm{Si}) .{ }^{13} \mathrm{C}$ NMR: $180.0(s, \mathrm{C}=0)$; $143.5(\mathrm{~s}, \mathrm{HC}=) ; 140.1$ (s, SiC=); 136.9 (s, arom. $\mathrm{C}) ; 128.4,128.2$ (2d, each 2 arom. $\mathrm{C}) ; 127.5$ (d, arom. C); $76.6\left(d, \mathrm{CF}_{3} \mathrm{CO}_{2} \mathrm{CH}\right) ; 26.9\left(q, \mathrm{Me}_{3} \mathrm{C}\right) ; 21.3(q, \mathrm{MeHC}=) ; 15.2\left(s, \mathrm{Me}_{3} C\right) ;-3.6,-4.1(2 q$, $\left.\mathrm{Me}_{2} \mathrm{Si}\right)$.

4.2. (Z)-2-[(tert-Butyl)dimethylsilyl]-1-phenylbut-2-enyl acetate (15b). Analogeously to 15a, $10 \mathrm{a}(600 \mathrm{mg}, 2.29 \mathrm{mmol})$, reacted with acetic anhydride $(351 \mathrm{mg}, 3.44 \mathrm{mmol})$ at reflux for $2 \mathrm{~h}$, gave after filtration through a plug of silica gel (hexane) $15 \mathrm{~b}(644 \mathrm{mg}, 2.12 \mathrm{mmol}, 93 \%)$ as a colorless oil. IR $\left(\mathrm{CHCl}_{3}\right): 3080 \mathrm{w}, 3060 \mathrm{w}, 3020 \mathrm{~m}, 2960 \mathrm{~s}, 2930 \mathrm{~s}, 2800 \mathrm{~m}, 2860 \mathrm{~s}, 1730 \mathrm{~s}, 1615 \mathrm{~m}, 1495 \mathrm{~m}$, $1475 \mathrm{~m}, 1465 \mathrm{~m}, 1455 \mathrm{~m}, 1410 \mathrm{w}, 1370 \mathrm{~s}, 1330 \mathrm{w}, 1245 \mathrm{~s}, 1220 \mathrm{~s}, 1180 \mathrm{w}, 1155 \mathrm{w}, 1130 \mathrm{~m}, 1080 \mathrm{w}$, $1060 \mathrm{~m}, 1015 \mathrm{~s}, 955 \mathrm{w}, 910 \mathrm{~m}, 835 \mathrm{~s}, 825 \mathrm{~s}, 700 \mathrm{~m}$. ${ }^{1} \mathrm{H}$ NMR: $7.32-7.25$ (m, 5 arom. H); 6.39 (q, HC=); $6.37\left(s, \mathrm{MeCO}_{2} \mathrm{CH}\right) ; 2.09\left(s, \mathrm{MeCO}_{2}\right) ; 1.87(d, J=6.7, \mathrm{MeHC}=) ; 0.90(s, t-\mathrm{Bu}) ; 0.08,0.02(2 s$, Mersi). ${ }^{13} \mathrm{C}$ NMR: 169.9 (s, C=0); 142.0 (d, HC=); 140.1 (s, SiC=); 136.5 (s, arom. C); $128.1,127.6$ (2d, each 2 arom. C); 127.5 (d, arom. C); $79.2\left(d, \mathrm{MeCO}_{2} \mathrm{CH}\right) ; 27.2\left(q, \mathrm{Me}_{3} \mathrm{C}\right) ; 21.3\left(q, \mathrm{MeCO}_{2}\right)$; $18.8\left(s, \mathrm{Me}_{3} \mathrm{C}\right) ; 18.6(q, \mathrm{MeHC}=) ;-3.4\left(q, \mathrm{Me}_{2} \mathrm{Si}\right)$. Cl-MS: $245\left(\left[\mathrm{M}+\mathrm{H}-\mathrm{MeCO}_{2} \mathrm{H}\right]^{+}\right)$.

4.3. (Z)-2-[(tert-Butyl)dimethylsilyl]-1-phenylbut-2-enyl chloroacetate (15c). Analogeously to $15 \mathrm{a}, 10 \mathrm{a}(528 \mathrm{mg}, 2.02 \mathrm{mmol})$, reacted with chloroacetic anhydride $(500 \mathrm{mg}, 2.92 \mathrm{mmol}$ ) in THF/ pyridine 5:1 and in the presence of 4-dimethylaminopyridine (cat.) at $23^{\circ} \mathrm{C}$ for $2 \mathrm{~h}$, gave after filtration through a plug of silica gel (hexane) $15 \mathrm{c}(627 \mathrm{mg}, 1.77 \mathrm{mmol}, 94 \%)$ as a colorless oil. IR $\left(\mathrm{CHCl}_{3}\right): 3090 w, 3060 w, 3030 w, 3010 w, 2960 s, 2930 s, 2900 \mathrm{~m}, 2880 \mathrm{~m}, 2860 \mathrm{~m}, 2860 \mathrm{~s}, 1750 \mathrm{~s}$, $1650 w, 1615 w, 1495 w, 1470 m, 1465 m, 1455 m, 1445 m, 1410 m, 1390 w, 1375 w, 1360 w, 1335 w$, $1305 \mathrm{~s}, 1260 \mathrm{~s}, 1170 \mathrm{~s}, 1130 \mathrm{~s}, 1100 \mathrm{~m}, 1080 \mathrm{~m}, 1060 \mathrm{~m}, 1010 \mathrm{~m}, 960 \mathrm{~m}, 895 \mathrm{w}, 835 \mathrm{~s}, 820 \mathrm{~s} .{ }^{1 \mathrm{H}}$ NMR: 7.34-7.26 (m, 5 arom. H); 6.50 (q, $\mathrm{HC}=) ; 6.44\left(\mathrm{~s}, \mathrm{H}_{2} \mathrm{ClCCO}_{2} \mathrm{CH}\right) ; 4.07$ (s, $\left.\mathrm{H}_{2} \mathrm{ClC}\right) ; 1.89$ (d, J= 7.4, MeHC=); 0.88 (s, $t$-Bu); 0.10, -0.01 (2s, Messi). ${ }^{13} \mathrm{C}$ NMR: $167.5(s, \mathrm{C}=0) ; 143.3$ (d, HC=); 140.4 (s, SiC=); 137.1 (s, arom. C); 129.6, 129.4 (2d, each 2 arom. C); 129.2 (d, arom. C); 82.4 (d, $\left.\mathrm{H}_{2} \mathrm{ClCCO}_{2} \mathrm{CH}\right) ; 42.5\left(t, \mathrm{H}_{2} \mathrm{ClC}\right) ; 28.5\left(q, \mathrm{Me}_{3} \mathrm{C}\right) ; 20.2\left(\mathrm{~s}, \mathrm{Me}_{3} \mathrm{C}\right) ; 20.0(t, \mathrm{MeCH}) ;-2.0$ (q, Me $\left.2 \mathrm{Si}\right) . \mathrm{Cl}-$ MS: $259\left(\left[\mathrm{M}+\mathrm{H}-\mathrm{H}_{2} \mathrm{ClCCO}_{2} \mathrm{H}\right]^{+}\right)$.

4.4. (E)-2-[(tert-Butyl)dimethylsilyl]-1-phenylbut-2-enyl acetate (15d). Analogeously to 15a, $10 \mathrm{~b}(500 \mathrm{mg}, 1.91 \mathrm{mmol})$, reacted with acetic anhydride $(292 \mathrm{mg}, 2.86 \mathrm{mmol})$ in the presence of 4dimethylaminopyridine (cat.) at reflux for $2 \mathrm{~h}$, gave after filtration through a plug of silica gel (hexane) 15d (430 mg, $1.41 \mathrm{mmol}, 74 \%$ ) as a colorless oil. IR: $3080 \mathrm{w}, 3060 \mathrm{w}, 3030 \mathrm{w}, 3000 \mathrm{w}, 2950 \mathrm{~s}$, $2920 \mathrm{~s}, 2890 \mathrm{~s}, 2850 \mathrm{~s}, 2250 \mathrm{w}, 1740 \mathrm{~s}, 1600 \mathrm{w}, 1490 \mathrm{w}, 1470 \mathrm{~m}, 1460 \mathrm{~m}, 1445 \mathrm{~m}, 1410 \mathrm{w}, 1390 \mathrm{w}$, $1370 \mathrm{~m}, 1230 \mathrm{~s}, 1200 \mathrm{w}, 1180 \mathrm{w}, 1150 \mathrm{w}, 1120 \mathrm{w}, 1075 \mathrm{w}, 1020 \mathrm{~m}, 980 \mathrm{w}, 940 \mathrm{w}, 935 \mathrm{w}, 910 \mathrm{~m}, 835 \mathrm{~s}$, 825s, $810 m, 770 m, 735 s, 700 s .{ }^{1} \mathrm{H}$ NMR: $7.33-7.26(m, 5$ arom. $\mathrm{H}) ; 6.97\left(\mathrm{~s}, \mathrm{MeCO}_{2} \mathrm{CH}\right) ; 6.23(q$, $\mathrm{HC}=$ ); $2.17\left(s, \mathrm{MeCO}_{2}\right) ; 1.89$ ( $\left.d, J=6.8, \mathrm{MeHC}=\right) ; 0.85$ (s, t-Bu); 0.04,-0.08 (2s, $\left.\mathrm{Me}_{2} \mathrm{Si}\right)$. ${ }^{13} \mathrm{C}$ NMR: $170.2(\mathrm{~s}, \mathrm{C}=\mathrm{O}) ; 140.6(d, \mathrm{HC}=) ; 140.0(\mathrm{~s}, \mathrm{SiC}=) ; 138.5$ (s, arom. C); 128.1, 127.1 (2d, each 2 arom. C); 126.3 (d, arom. C); $74.4\left(d, \mathrm{MeCO}_{2} \mathrm{CH}\right) ; 26.9\left(q, \mathrm{Me}_{3} \mathrm{C}\right) ; 21.4\left(q, \mathrm{MeCO}_{2}\right) ; 17.3\left(\mathrm{~s}, \mathrm{Me}_{3} \mathrm{C}\right) ; 15.8$ $(q, \mathrm{MeCH}) ;-3.8,-4.6\left(2 q, \mathrm{Me}_{2} \mathrm{Si}\right) . \mathrm{Cl}-\mathrm{MS}: 245\left(\left[\mathrm{M}+\mathrm{H}-\mathrm{MeCO}_{2} \mathrm{H}\right]^{+}\right)$.

4.5. (Z)-2-\{(tert-Butyl)dimethylsilyl\}-1-ethyl-3-phenylprop-2-enyl acetate (15e). Analogeously to $15 \mathrm{a}, 10 \mathrm{c}(200 \mathrm{mg}, 0.72 \mathrm{mmol})$, reacted with acetic anhydride $(111 \mathrm{mg}, 1.09 \mathrm{mmol})$ in the presence of 4-dimethylaminopyridine (cat.) at reflux for $2 \mathrm{~h}$, gave after filtration through a plug of silica gel (hexane) $15 \mathrm{e}(191 \mathrm{mg}, 0.60 \mathrm{mmol}, 83 \%)$ as a colorless oil. IR $\left(\mathrm{CHCl}_{3}\right): 3080 \mathrm{w}, 3050 \mathrm{w}, 3020 \mathrm{w}$, $2960 \mathrm{~s}, 2930 \mathrm{~s}, 2880 \mathrm{~s}, 2850 \mathrm{~s}, 1740 \mathrm{~s}, 1590 \mathrm{~m}, 1575 \mathrm{w}, 1490 \mathrm{~m}, 1460 \mathrm{~m}, 1440 \mathrm{~m}, 1405 \mathrm{~m}, 1365 \mathrm{~s}$, $1235 \mathrm{~s}, 1130 \mathrm{w}, 1090 \mathrm{~m}, 1070 \mathrm{w}, 1030 \mathrm{~s}, 1015 \mathrm{~s}, 955 \mathrm{~m}, 935 \mathrm{w}, 910 \mathrm{w}, 895 \mathrm{w}, 860 \mathrm{w}, 835 \mathrm{~s}, 820 \mathrm{~s}$, $810 s, 770 s, 750 s, 730 w, 700 s, 685 w .{ }^{1} \mathrm{H}$ NMR: $7.66(s, \mathrm{HC}=) ; 7.36-7.22(\mathrm{~m}, 5 \mathrm{arom} . \mathrm{H}) ; 4.81$ (dd, $\left.J=8.3,3.5, \mathrm{MeCO}_{2} \mathrm{CH}\right) ; 2.19\left(s, \mathrm{MeCO}_{2}\right) ; 1.96-1.68(\mathrm{~m}, \mathrm{MeCH}) ; 1.03\left(t, J=7.4, \mathrm{MeCH}_{2}\right) ; 0.96$ (s, t-Bu);0.02, -0.13 (2s, Me $2 \mathrm{Si}) .{ }^{13} \mathrm{C}$ NMR: $170.2(s, \mathrm{C}=0) ; 143.5(d, \mathrm{HC}=) ; 141.4(s, \mathrm{SiC}=) ; 140.0$ (s, arom. C); 128.4, 127.5 (2d, each 2 arom. C); 126.8 (d, arom. C); $77.5\left(d, \mathrm{MeCO}_{2} \mathrm{CH}\right) ; 29.6(t$, $\left.\mathrm{MeCH}_{2}\right) ; 27.5\left(q, \mathrm{Me}_{3} \mathrm{C}\right) ; 21.3\left(q, \mathrm{MeCO}_{2}\right) ; 17.9\left(s, \mathrm{Me}_{3} \mathrm{C}\right) ; 10.4\left(q, \mathrm{MeCH}_{2}\right) ;-3.0,-3.1(2 q$, $\left.\mathrm{Me}_{2} \mathrm{Si}\right)$. Cl-MS: $259\left(\left[\mathrm{M}+\mathrm{H}-\mathrm{MeCO}_{2} \mathrm{H}\right]^{+}\right)$.

4.6. (Z)-2-\{(tert-Butyl)dimethylsilyl\}-1-ethyl-3-phenylprop-2-enyl chloroacetate (15f). Analogeously to $15 \mathrm{a}, 10 \mathrm{c}(400 \mathrm{mg}, 1.44 \mathrm{mmol})$, reacted with chloroacetic anhydride ( $370 \mathrm{mg}, 2.17$ $\mathrm{mmol}$ ) in THF/pyridine 5:1 and in the presence of 4-dimethylaminopyridine (cat.) at $23^{\circ} \mathrm{C}$ for $2 \mathrm{~h}$, gave after filtration through a plug of silica gel (hexane) $15 f(495 \mathrm{mg}, 1.40 \mathrm{mmol}, 97 \%)$ as a colorless oil. IR: $3080 w, 3050 w, 3020 w, 2960 s, 2930 s, 2880 m, 2850 s, 1760 s, 1740 s, 1590 w, 1490 w$, $1460 \mathrm{~m}, 1440 \mathrm{w}, 1410 \mathrm{w}, 1390 \mathrm{w}, 1380 \mathrm{w}, 1360 \mathrm{w}, 1290 \mathrm{~s}, 1260 \mathrm{~s}, 1185 \mathrm{~s}, 1130 \mathrm{~m}, 1090 \mathrm{~m}, 1070 \mathrm{w}$, $1025 \mathrm{~m}, 1010 \mathrm{w}, 960 \mathrm{~m}, 925 \mathrm{w}, 910 \mathrm{w}, 895 \mathrm{w}, 860 \mathrm{w}, 835 \mathrm{~s}, 820 \mathrm{~s}, 810 \mathrm{~s}, 770 \mathrm{~s}, 750 \mathrm{~s}, 730 \mathrm{w}, 700 \mathrm{~s} .{ }^{1} \mathrm{H}$ NMR: $7.70(\mathrm{~s}, \mathrm{HC}=) ; 7.38-7.24(\mathrm{~m}, 5$ arom. $\mathrm{H}) ; 5.65-5.57\left(\mathrm{~m}, \mathrm{H}_{2} \mathrm{ClCCO}_{2} \mathrm{CH}\right) ; 4.20\left(\mathrm{~s}, \mathrm{H}_{2} \mathrm{ClC}\right)$; 
2.11-1.79 (m, MeCH$\left.H_{2}\right) ; 1.09$ (q, $t$-Bu); $0.97\left(t, J=7.4, \mathrm{MeCH}_{2}\right) ; 0.04,-0.10$ (2s, Mes Si). ${ }^{13} \mathrm{C} \mathrm{NMR:}$ $166.5(\mathrm{~s}, \mathrm{C}=\mathrm{O})$; 143.9 (d, $\mathrm{HC}=) ; 140.8(\mathrm{~s}, \mathrm{SiC}=) ; 137.7$ (s, arom. $\mathrm{C}) ; 128.4,127.5$ (2d, each 2 arom. C); 127.0 (d, arom. C); 79.7 (d, $\left.\mathrm{H}_{2} \mathrm{ClCCO}_{2} \mathrm{CH}\right) ; 41.2\left(t, \mathrm{H}_{2} \mathrm{ClC}\right) ; 29.7\left(t, \mathrm{MeCH}_{2}\right) ; 27.5$ (q, Mes $\mathrm{M}$; 17.9 (s, Mes $\mathrm{C}) ; 10.4\left(q, \mathrm{MeCH}_{2}\right) ;-3.1,-3.2\left(2 q, \mathrm{Me}_{2} \mathrm{Si}\right)$. Cl-MS: $245\left(\left[\mathrm{M}+\mathrm{H}-\mathrm{H}_{2} \mathrm{ClCO}_{2} \mathrm{H}\right]^{+}\right)$.

4.7. (Z)-2-\{(tert-Butyl)dimethylsilyl\}-1-ethyl-3-phenylprop-2-enyl dichloroacetate (15g). Analogeously to $15 \mathrm{a}, 10 \mathrm{c}$ (300 mg, $1.09 \mathrm{mmol})$, reacted with dichloroacetyl chloride (240 mg, 1.63 $\mathrm{mmol}$ ) in THF in the presence of pyridine $(0.12 \mathrm{ml}, 1.63 \mathrm{mmol})$ and 4-dimethylaminopyridine (cat.) at $23^{\circ} \mathrm{C}$ for $1 \mathrm{~h}$, gave after filtration through a plug of silica gel (hexane) $15 \mathrm{~g}(387 \mathrm{mg}, 1.00 \mathrm{mmol}$, $92 \%)$ as a colorless oil. IR $\left(\mathrm{CHCl}_{3}\right): 3080 w, 3060 w, 3020 w, 2960 \mathrm{~s}, 2930 \mathrm{~s}, 2880 \mathrm{~m}, 2860 \mathrm{~s}, 1755 \mathrm{~s}$, $1590 w, 1490 w, 1465 m, 1440 w, 1405 w, 1390 w, 1360 w, 1290 s, 1280 s, 1240 s, 1220 w, 1170 s$, $1115 w, 1090 w, 1070 w, 1030 w, 1010 w, 910 w, 900 w, 860 w, 835 s, 820 s, 700 m$. ${ }^{1} \mathrm{H}$ NMR: 7.75 (s, $\mathrm{HC}=$ ); 7.38, $7.23(\mathrm{~m}, 5$ arom. $\mathrm{H}) ; 6.09\left(\mathrm{~s}, \mathrm{HCl}_{2} \mathrm{C}\right) ; 5.63-5.62\left(\mathrm{~m}, \mathrm{HCl}_{2} \mathrm{CO}_{2} \mathrm{CH}\right) ; 2.71-2.52$ (m, $\left.\mathrm{MeCH}_{2}\right) ; 1.13\left(t, J=7.4, \mathrm{MeCH}_{2}\right) ; 0.97(s, t-\mathrm{Bu}) ; 0.04,-0.09$ (2s, Me $\left.2 \mathrm{Si}\right) .{ }^{13} \mathrm{C} \mathrm{NMR:} 163.6(s, \mathrm{C}=\mathrm{O})$; 144.2 (d, HC=); 140.3 (s, SiC=); 139.5 (s, arom. C); 128.3, 127.5 (2d, each 2 arom. C); 127.0 (d, arom. C); $81.2\left(d, \mathrm{HCl}_{2} \mathrm{CCO}_{2} \mathrm{CH}\right) ; 64.7\left(d, \mathrm{HCl}_{2} \mathrm{C}\right) ; 29.8\left(t, \mathrm{MeCH}_{2}\right) ; 27.4\left(q, \mathrm{Me}_{3} \mathrm{C}\right) ; 17.9$ (s, $\mathrm{Me}_{3} \mathrm{C}$ ); $10.4\left(q, \mathrm{MeCH}_{2}\right) ;-3.20,-3.22\left(2 q, \mathrm{Me}_{2} \mathrm{Si}\right) . \mathrm{Cl}-\mathrm{MS}: 259\left(\left[\mathrm{M}+\mathrm{H}-\mathrm{HCl}_{2} \mathrm{CCO}_{2} \mathrm{H}\right]^{+}\right)$.

4.8. (E)-2-\{(tert-Butyl)dimethylsilyl\}-1-ethyl-3-phenylprop-2-enyl chloroacetate (15h). Analogeously to $15 \mathrm{a}, 10 \mathrm{~d}(436 \mathrm{mg}(1.58 \mathrm{mmol})$, reacted with chloroacetic anhydride (405 $\mathrm{mg}, 2.37$ $\mathrm{mmol}$ ) in THF/pyridine 5:1 and in the presence of 4-dimethylaminopyridine (cat.) at $23^{\circ} \mathrm{C}$ for $2 \mathrm{~h}$, gave after filtration through a plug of silica gel (hexane) $15 \mathrm{~h}(535 \mathrm{mg}, 1.59 \mathrm{mmol}, 96 \%)$ as a colorless oil. IR: $3070 w, 3050 w, 3020 w, 2950 s, 2920 s, 2880 m, 2850 s, 1755 s, 1730 s, 1590 w$, $1570 \mathrm{w}, 1490 \mathrm{w}, 1460 \mathrm{~m}, 1440 \mathrm{w}, 1410 \mathrm{~m}, 1390 \mathrm{w}, 1350 \mathrm{w}, 1360 \mathrm{~m}, 1305 \mathrm{~s}, 1280 \mathrm{~s}, 1250 \mathrm{~s}, 1180 \mathrm{~s}$, $1135 \mathrm{~m}, 1075 \mathrm{~m}, 1025 \mathrm{w}, 1010 \mathrm{~m}, 960 \mathrm{~s}, 925 \mathrm{~m}, 900 \mathrm{w}, 835 \mathrm{~s}, 820 \mathrm{~s}, 810 \mathrm{~s}, 770 \mathrm{~s}, 700 \mathrm{~s}$. ${ }^{1} \mathrm{H}$ NMR: 7.39-7.25 (m, 5 arom. H); 6.99 (s, $\mathrm{HC}=)$; 6.00-5.90 ( $\left.m, \mathrm{H}_{2} \mathrm{ClCO}_{2} \mathrm{CH}\right) ; 4.00,3.91$ ( $A B, J=14.6$, $\left.\mathrm{H}_{2} \mathrm{ClC}\right) ; 1.97-1.69,1.68-1.50\left(2 \mathrm{~m}, \mathrm{MeCH}_{2}\right) ; 1.00(\mathrm{~s}, \mathrm{t}-\mathrm{Bu}) ; 0.86\left(t, J=7.4, \mathrm{MeCH}_{2}\right) ; 0.30,0.26$ $\left(2 s, \mathrm{Me}_{2} \mathrm{Si}\right) .{ }^{13} \mathrm{C}$ NMR: $166.6(s, \mathrm{C}=0) ; 143.0$ (d, $\left.\mathrm{HC}=\right) ; 140.5$ (s, SiC=); 137.8 (s, arom. C); 128.3, 128.2 (2d, each 2 arom. C); 127.0 (d, arom. C); $78.9\left(d, \mathrm{H}_{2} \mathrm{ClCCO}_{2} \mathrm{CH}\right) ; 41.0\left(t, \mathrm{H}_{2} \mathrm{ClC}\right) ; 28.7$ (t, $\mathrm{MeCH}_{2}$ ); 27.0 (q, Mes $\mathrm{C}$ ); $17.6\left(\mathrm{~s}, \mathrm{Me}_{3} \mathrm{C}\right) ; 10.2$ (q, $\left.\mathrm{MeCH}_{2}\right) ;-3.5,-3.9$ (2q, Me $\left.2 \mathrm{Si}\right)$. Cl-MS: 259 ([M $\left.+\mathrm{H}-\mathrm{H}_{2} \mathrm{ClCO}_{2} \mathrm{H}\right]^{+}$).

4.9. (Z)-2-[(tert-Butyl)dimethylsilyl]-1,3-diphenylprop-2-enyl acetate (15i). Analogeously to $15 \mathrm{a}, 10 \mathrm{e}$ (600 mg, $1.85 \mathrm{mmol})$, reacted with acetic anhydride $(284 \mathrm{mg}, 2.77 \mathrm{mmol})$ in the presence of 4-dimethylaminopyridine (cat.) at reflux for $2 \mathrm{~h}$, gave after filtration through a plug of silica gel (hexane) $15 \mathrm{i}(598 \mathrm{mg}, 1.63 \mathrm{mmol}, 88 \%)$ as a colorless oil. IR $\left(\mathrm{CHCl}_{3}\right): 3080 \mathrm{w}, 3060 \mathrm{w}, 3030 \mathrm{w}$, $3010 w, 2960 s, 2930 s, 2890 m, 2860 s, 1730 s, 1590 m, 1490 m, 1470 m, 1465 m, 1410 w, 1390 w$, $1370 s, 1240 s, 1220 s, 1180 w, 1070 w, 1050 w, 1030 m, 1025 s, 960 m, 915 w, 890 w, 865 w, 840 s$, 825s, 810s, 700s. ${ }^{1} \mathrm{H}$ NMR: 7.63 (s, HC=); 7.48-7.24 (m, 10 arom. $\left.\mathrm{H}\right) ; 6.64\left(\mathrm{~s}, \mathrm{MeCO}_{2} \mathrm{CH}\right) ; 2.15$ ( $\mathrm{s}$, $\left.\mathrm{MeCO}_{2}\right) ; 0.87$ (s, t-Bü); $-0.20,-0.27\left(2 s, \mathrm{Me}_{2} \mathrm{Si}\right) .{ }^{13} \mathrm{C} \mathrm{NMR:} 169.8(s, \mathrm{C}=0) ; 145.4$ (d, HC=); 140.0 $(s, \mathrm{SiC}=$ ); 138.8 (s, arom. C); 139.6 (s, arom. C); 128.5, 128.3 (2d, each 2 arom. C); 128.2 (d, arom. C); 128.0, 127.5 (2d, each 2 arom. C); 126.9 (d, arom. C); 78.1 (d, $\left.\mathrm{MeCO}_{2} \mathrm{CH}\right) ; 27.6\left(q, \mathrm{Me}_{3} \mathrm{C}\right)$; $21.4\left(q, \mathrm{MeCO}_{2}\right) ; 18.0\left(s_{,} \mathrm{Me}_{3} \mathrm{C}\right) ;-2.9\left(q, \mathrm{Me}_{2} \mathrm{Si}\right) . \mathrm{Cl}-\mathrm{MS}: 307\left(\left[\mathrm{M}+\mathrm{H}-\mathrm{MeCO}_{2} \mathrm{H}\right]^{+}\right)$.

4.10. (E)-2-[(tert-Butyl)dimethylsilyl]-1,3-diphenylprop-2-enyl acetate (15j). Analogeously to $15 \mathrm{a}, 10 \mathrm{f}(260 \mathrm{mg}, 0.80 \mathrm{mmol})$, reacted with acetic anhydride $(94 \mathrm{mg}, 0.93 \mathrm{mmol}$ ) in the presence of 4-dimethylaminopyridine (cat.) at reflux for $2 \mathrm{~h}$, gave after filtration through a plug of silica gel (hexane) 15j (239 mg, $0.65 \mathrm{mmol}, 81 \%)$ as a colorless oil. IR $\left(\mathrm{CHCl}_{3}\right): 3080 \mathrm{w}, 3060 \mathrm{w}, 3030 \mathrm{w}$, $3000 \mathrm{w}, 2950 \mathrm{~s}, 2930 \mathrm{~s}, 2880 \mathrm{~m}, 2850 \mathrm{~s}, 1730 \mathrm{~s}, 1600 \mathrm{w}, 1570 \mathrm{w}, 1490 \mathrm{w}, 1460 \mathrm{w}, 1440 \mathrm{w} .1405 \mathrm{w}$, $1370 m, 1240 s, 1100 m, 1070 m, 1015 s, 970 m, 940 w, 920 w, 905 w, 885 w, 820 s, 695 s .{ }^{1} H$ NMR: 7.33-7.24 (m, 10 arom. H); $7.17(s, \mathrm{HC}=) ; 7.11\left(s, \mathrm{MeCO}_{2} \mathrm{CH}\right) ; 2.08\left(\mathrm{~s}, \mathrm{MeCO}_{2}\right) ; 0.94$ (s, $\left.t-\mathrm{Bu}\right)$; $0.07,-0.12\left(2 s, \mathrm{Me}_{2} \mathrm{Si}\right) .{ }^{13} \mathrm{C}$ NMR: $170.0(\mathrm{~s}, \mathrm{C}=0) ; 143.7(d, \mathrm{HC}=) ; 139.9(s, \mathrm{SiC}=) ; 139.6$ ( $s$, arom. C); 137.3 (s, arom. C); 128.3, 128.2 (2d, each 2 arom. C); 127.3 (d, arom. C); 127.2 (d, 2 arom. C); 126.3 (d, 3 arom. C); 74.7 (d, $\mathrm{MeCO}_{2} \mathrm{CH}$ ); 27.0 (q, Mes $\left.\mathrm{C}\right) ; 21.3\left(q, \mathrm{MeCO}_{2}\right) ; 17.6\left(\mathrm{~s}, \mathrm{Me}_{3} \mathrm{C}\right) ;-3.3$, $-4.7\left(2 q, \mathrm{Me}_{2} \mathrm{Si}\right)$. $\mathrm{Cl}-\mathrm{MS}: 307\left(\left[\mathrm{M}+\mathrm{H}-\mathrm{MeCO}_{2} \mathrm{H}\right]^{+}\right)$.

4.11. (Z)-2-\{(tert-Butyl)dimethylsilyl\}-1-isopropyl-3-phenylprop-2-enyl dichloroacetate (15k). Analogeously to $15 \mathrm{a}, 10 \mathrm{~g}$ (300 mg, $1.03 \mathrm{mmol}$ ), reacted with dichloroacetyl chloride $(226 \mathrm{mg}$, $1.55 \mathrm{mmol})$ in THF $(2 \mathrm{ml})$ and pyridine $(0.16 \mathrm{ml}, 2.0 \mathrm{mmol})$ and in the presence of 4-dimethylaminopyridine (cat.) at $23^{\circ} \mathrm{C}$ for $1 \mathrm{~h}$, gave after filtration through a plug of silica gel (hexane) $15 \mathrm{~K}$ (375 mg, $0.94 \mathrm{mmol}, 90 \%)$ as a colorless oil. ${ }^{1} \mathrm{H}$ NMR: $7.38-7.26(\mathrm{~m}, 5$ arom. $\mathrm{H}) ; 7.09(\mathrm{~s}, \mathrm{HC}=) ; 5.77(d, J=$ 
9.9, $\left.\mathrm{HCl}_{2} \mathrm{CCO}_{2} \mathrm{CH}\right) ; 2.05-1.93\left(m, \mathrm{Me}_{2} \mathrm{CH}\right) ; 0.96(s, t-\mathrm{Bu}) ; 0.88,0.72\left(2 d, J=6.5,6.9, \mathrm{Me}_{2} \mathrm{CH}\right)$; 0.26 (s, Me $2 \mathrm{Si})$. Cl-MS: $418\left(2,\left[\mathrm{M}+\mathrm{NH}_{4}\right]^{+}\right), 273\left(100,\left[\mathrm{M}+\mathrm{H}-\mathrm{HCl}_{2} \mathrm{CCO}_{2} \mathrm{H}\right]^{+}\right)$.

4.12. (Z)-2-(tert-Butyl)dimethylsilyl\}-1.5-diphenylpent-2-enyl acetate (15I). Analogeously to $15 \mathrm{a}, 10 \mathrm{~h}(400 \mathrm{mg}, 1.14 \mathrm{mmol})$, reacted with acetic anhydride $(176 \mathrm{mg}, 1.70 \mathrm{mmol})$ in the presence of 4-dimethylaminopyridine (cat.) at reflux for $2 \mathrm{~h}$, gave after filtration through a plug of silica gel (hexane) 151 (400 mg, $1.02 \mathrm{mmol}, 89 \%$ ) as a colorless oil. IR (film): $3080 \mathrm{w}, 3060 \mathrm{w}, 3030 \mathrm{~m}$, $2950 s, 2930 s, 2900 m, 2850 s, 1950 w, 1740 s, 1605 m, 1385 w, 1490 m, 1470 m, 1460 m, 1450 m$, $1410 w, 1390 w, 1370 s, 1235 w, 1250 s, 1230 s, 1180 w, 1155 w, 1055 m, 1030 m, 1025 s, 955 m$, $940 w, 910 w, 835 s, 820 s, 810 s, 775 m, 760 m, 750 s, 700 s .{ }^{1} \mathrm{H}$ NMR: $7.40-7.23(m, 11 \mathrm{H}, 10$ arom, $\mathrm{H}$, and $\left.\mathrm{MeCO}_{2} \mathrm{CH}\right) ; 6.45(t, J=7.7, \mathrm{HC}=) ; 2.87-2.76\left(m, \mathrm{PhCH}_{2}\right) ; 2.71-2.60\left(m, \mathrm{CH}_{2} \mathrm{C}=\right) ; 2.17(\mathrm{~s}$, $\mathrm{MeCO}_{2}$ ); 0.96 (s, t-Bu); 0.13, 0.04 (2s, Mersi). ${ }^{13} \mathrm{C} \mathrm{NMR:} 169.8$ ( $\left.s, \mathrm{C}=0\right) ; 146.2$ (d, HC=); $141.4(s$, $\mathrm{SiC}=$ ); 140.0 (s, arom. C); 136.0 (s, arom. C); $128.5,128.3$ (2d, each 2 arom. C); 128.1 (d, arom. C); 127.7, 127.6 (2d, each 2 arom. C); 125.9 (d, arom. C); $79.1\left(d, \mathrm{MeCO}_{2} \mathrm{CH}\right) ; 36.0\left(t, \mathrm{PhCH}_{2}\right) ; 34.7(t$, $\left.\mathrm{CH}_{2} \mathrm{C}=\right) ; 27.2\left(q, \mathrm{Me}_{3} \mathrm{C}\right) ; 21.3\left(q, \mathrm{MeCO}_{2}\right) ; 18.3\left(s, \mathrm{Me}_{3} \mathrm{C}\right) ;-3.3\left(q, \mathrm{Me}_{2} \mathrm{Si}\right)$. Cl-MS: $335([\mathrm{M}+\mathrm{H}-$ $\left.\left.\mathrm{MeCO}_{2} \mathrm{H}\right]^{+}\right)$.

4.13. (Z)-2-(tert-Butyl)dimethylsilyl\}-1.5-diphenylpent-2-enyl chloroacetate (15m). Analogeously to $15 \mathrm{a}, 10 \mathrm{~h}(300 \mathrm{mg}, 0.85 \mathrm{mmol})$, reacted with chloroacetic anhydride (218 $\mathrm{mg}, 1.28$ $\mathrm{mmol}$ ) in THF/pyridine 5:1 and in the presence of 4-dimethylaminopyridine (cat.) at $23^{\circ} \mathrm{C}$ for $2 \mathrm{~h}$, gave after filtration through a plug of silica gel (hexane) $15 \mathrm{~m}(310 \mathrm{mg}, 0.72 \mathrm{mmol}, 85 \%$ ) as a colorless oil. IR: $3080 w, 3060 w, 3020 w, 2950 s, 2920 s, 2990 m, 2850 s, 1760 s, 1735 s, 1600 w, 1490 w$, $1470 w, 1460 w, 1450 m, 1410 w, 1385 w, 1360 w, 1335 w, 1305 m, 1280 m, 1255 s, 1250 s, 1165 s$, $1130 m, 1055 m, 1030 w, 1010 w, 960 m, 910 w, 835 s, 820 s, 810 m, 790 w, 760 m, 745 m, 700 s .{ }^{1} \mathrm{H}$ NMR: $7.35-7.19(m, 10$ arom. $\mathrm{H}) ; 6.47(t, J=6.0, \mathrm{HC}=) ; 6.45\left(s, \mathrm{H}_{2} \mathrm{ClCCO}_{2} \mathrm{CH}\right) ; 4.08\left(s_{1} \mathrm{H}_{2} \mathrm{ClC}\right)$; 2.86-2.69 $\left(m, \mathrm{PhCH}_{2}\right) ; 2.69-2.53\left(m, \mathrm{CH}_{2} \mathrm{C}=\right) ; 0.89(s, t-\mathrm{Bu}) ; 0.10,-0.04\left(2 s, \mathrm{Me}_{2} \mathrm{Si}\right),{ }^{13} \mathrm{C}$ NMR: $166.1(\mathrm{~s}, \mathrm{C}=0) ; 145.9$ (s, HC=); $141.2(\mathrm{~s}, \mathrm{SiC}=) ; 138.8$ (s, arom. C); 135.2 (s, arom. C); 128.5, 128.3 ( $2 d$, each 2 arom. C); 128.2 ( $d$, arom. C); $128.1,128.0$ (2d, each 2 arom. C); 125.9 ( $d$, arom. C); $80.8\left(d, \mathrm{H}_{2} \mathrm{ClCCO}_{2} \mathrm{CH}\right) ; 41.1\left(t, \mathrm{H}_{2} \mathrm{ClC}\right) ; 36.0\left(t, \mathrm{PhCH}_{2}\right) ; 34.6\left(t, \mathrm{CH}_{2} \mathrm{C}=\right) ; 27.1\left(q, \mathrm{Me}_{3} \mathrm{C}\right) ; 18.3$ $\left(s, \mathrm{Me}_{3} \mathrm{C}\right) ;-3.4(q, \mathrm{Me} 2 \mathrm{Si}) . \mathrm{Cl}-\mathrm{MS}: 335\left(\left[\mathrm{M}+\mathrm{H}-\mathrm{H}_{2} \mathrm{ClCCO}_{2} \mathrm{H}\right]^{+}\right)$.

4.14. (Z)-2-\{[(Benzyloxy)methyl](tert-butyl)methylsilyl\}-1-phenylbut-2-enyl acetate (16a). Analogeously to $15 \mathrm{a}, 11 \mathrm{a}(150 \mathrm{mg}, 0.41 \mathrm{mmol})$, reacted with acetic anhydride $(60 \mathrm{mg}, 0.57 \mathrm{mmol})$ in the presence of 4-dimethylaminopyridine (cat.) at reflux for $2 \mathrm{~h}$, gave after filtration through a plug of silica gel (hexane) $16 a(140 \mathrm{mg}, 0.34 \mathrm{mmol}, 86 \%)$ as a colorless oil. IR: $3080 \mathrm{w}, 3060 \mathrm{w}, 3000 \mathrm{~m}$, $2930 s, 2840 s, 1730 s, 1615 w, 1495 m, 1460 m, 1450 m, 1370 s, 1240 s, 1090 s, 1070 s, 1015 s$, $955 m, 900 w, 825 s, 700 s$. $1 \mathrm{H}$ NMR: $7.29-7.25(m, 10$ arom. H); $6.40(q, J=6.4, H C=) ; 6.35(s$, $\left.\mathrm{MeCO}_{2} \mathrm{CH}\right) ; 4.39\left(\mathrm{~s}, \mathrm{PhCH}_{2} \mathrm{O}\right) ; 3.31$, $3.34\left(\mathrm{AB}, J=12.8, \mathrm{SiCH}_{2} \mathrm{O}\right) ; 1.82(d, J=7.3, \mathrm{MeCH}) ; 0.88(s$, $t$-Bu); 0.05 (s, MeSi). ${ }^{13} \mathrm{C}$ NMR: $168.4(s, \mathrm{C}=0) ; 141.0(d, \mathrm{HC}=)$; $138.0(\mathrm{~s}, \mathrm{SiC}=), 137.5$ (s, arom. $\mathrm{H}$ ); 133.8 ( $s$, arom. C); 126.8, 126.6 (2d, each 2 arom. C); 126.3 (d, arom. C), 126.2 (d, 2 arom. C); 125.9 (d, 3 arom. C); $77.2\left(d, \mathrm{MeCO}_{2} \mathrm{CH}\right) ; 75.8\left(t, \mathrm{PhCH}{ }_{2} \mathrm{O}\right) ; 60.6\left(t, \mathrm{SiCH}_{2} \mathrm{O}\right) ; 26.3\left(q, \mathrm{Me}_{3} \mathrm{C}\right) ; 20.0$ $\left(q, \mathrm{MeCO}_{2}\right) ; 17.6(q, \mathrm{MeCH}) ; 17.4\left(\mathrm{~s}, \mathrm{Me}_{3} \mathrm{C}\right) ;-7.7$ (q, MeSi). CI-MS: $428\left(20,\left[\mathrm{M}+\mathrm{NH}_{4}\right]^{+}\right), 351(100$, $\left.\left[\mathrm{M}+\mathrm{H}-\mathrm{MeCO}_{2} \mathrm{H}\right]^{+}\right)$.

4.15. (E)-2-\{[(Benzyloxy)methyl](tert-butyl)methylsilyl\}-1-phenylbut-2-enyl acetate (16b). Analogeously to $15 \mathrm{a}, 11 \mathrm{c}(330 \mathrm{mg}, 0.90 \mathrm{mmol}$ ), reacted with acetic anhydride (138 $\mathrm{mg}, 1.35$ $\mathrm{mmol}$ ) in the presence of 4-dimethylaminopyridine (cat.) at reflux for $2 \mathrm{~h}$, gave after filtration through a plug of silica gel (hexane) $16 \mathrm{~b}(300 \mathrm{mg}, 0.73 \mathrm{mmol}, 82 \%)$ as a colorless oil. Optically active (+)$16 \mathrm{~b}$ was obtained likewise from $(-)-11 \mathrm{c} .(+)-16 \mathrm{~b}:[\alpha]^{23}=+8(\mathrm{c}=0.9$, THF). IR: $3080 \mathrm{w}, 3060 \mathrm{w}$, $3020 m, 2950 s, 2920 s, 2880 m, 2850 s, 2810 m, 1945 w, 1800 w, 1740 s, 1600 m, 1580 w, 1490 m$, $1470 \mathrm{~m}, 1460 \mathrm{~m}, 1450 \mathrm{~m}, 1430 \mathrm{~m}, 1370 \mathrm{~s}, 1230 \mathrm{~s}, 1200 \mathrm{~m}, 1180 \mathrm{w}, 1150 \mathrm{w}, 1090 \mathrm{~m}, 1070 \mathrm{~s}, 1020 \mathrm{~s}$, $1000 \mathrm{~m}, 980 \mathrm{~m}, 955 \mathrm{~m}, 935 \mathrm{w}, 905 \mathrm{w}, 830 \mathrm{~s}, 785 \mathrm{~m}, 770 \mathrm{~m}, 750 \mathrm{~s}, 735 \mathrm{~s}, 700 \mathrm{~s} .{ }^{1} \mathrm{H}$ NMR: $7.32-7.25(\mathrm{~m}$, 10 arom. H); $6.90(q, \mathrm{HC}=) ; 4.37\left(\mathrm{~s}, \mathrm{PhCH}_{2} \mathrm{O}\right) ; 3.28,3.13\left(\mathrm{AB}, J=12.8, \mathrm{SiCH}_{2} \mathrm{O}\right) ; 2.07\left(\mathrm{~s}, \mathrm{MeCO}_{2}\right)$; $1.84(d, J=6.8, \mathrm{MeHC}=) ; 0.87(s, t-\mathrm{Bu}) ; 0.12(s, \mathrm{MeSi}) .{ }^{13} \mathrm{C} \mathrm{NMR}: 170.0(s, \mathrm{C}=0) ; 141.3(d, \mathrm{HC}=) ;$ 139.8 (s, SiC=); 139.0 (s, arom. C); 137.1 (s, arom. C); $128.1,128.0$ (2d, each 2 arom. C); 127.4 (d, arom. C); 127.2, 127.1 (2d, each 2 arom. C); 126.7 (d, arom. C); $76.9\left(t, \mathrm{PhCH}_{2} \mathrm{O}\right) ; 74.4$ (d, $\left.\mathrm{MeCO}_{2} \mathrm{CH}\right) ; 61.6\left(t, \mathrm{SiCH}_{2} \mathrm{O}\right) ; 27.3\left(q, \mathrm{Me}_{3} \mathrm{C}\right) ; 21.3\left(q, \mathrm{MeCO}_{2}\right) ; 17.5\left(s, \mathrm{Me}_{3} \mathrm{C}\right) ; 16.1(q, \mathrm{MeHC}=)$; $-6.9(q, \mathrm{MeSi}) . \mathrm{Cl}-\mathrm{MS}: 351\left(\left[\mathrm{M}+\mathrm{NH}_{4}\right]^{+}\right)$.

4.16. (Z)-2-\{[(Benzyloxy)methyl](tert-butyl)methy/silyl\}-1-isopropylbut-2-enyl acetate (16c). Analogeously to $15 \mathrm{a}, 11 \mathrm{f}(170 \mathrm{mg}, 0.51 \mathrm{mmol})$, reacted with acetic anhydride $(104 \mathrm{mg}, 1.02$ $\mathrm{mmol}$ ) in the presence of 4-dimethylaminopyridine (cat.) at reflux for $2 \mathrm{~h}$, gave after filtration through 
a plug of silica gel (hexane) $16 \mathrm{c}(182 \mathrm{mg}, 0.48 \mathrm{mmol}, 95 \%)$ as a colorless oil. IR $\left(\mathrm{CHCl}_{3}\right): 3080 \mathrm{w}$, $3060 w, 3020 w, 2960 s, 2930 s, 2890 s, 2850 s, 2820 w, 1725 s, 1610 w, 1490 w, 1460 m, 1450 m$, $1430 \mathrm{~m}, 1365 \mathrm{~m}, 1270 \mathrm{~m}, 1250 \mathrm{~s}, 1220 \mathrm{~s}, 1140 \mathrm{w}, 1090 \mathrm{~m}, 1070 \mathrm{~m}, 1050 \mathrm{w}, 1020 \mathrm{~m}, 980 \mathrm{w}, 955 \mathrm{w}$, $940 \mathrm{w}, 930 \mathrm{w}, 900 \mathrm{w}, 820 \mathrm{~m} .{ }^{1} \mathrm{H}$ NMR: $7.35-7.24(\mathrm{~m}, 5$ arom. $\mathrm{H}) ; 6.39$ ( $\left.q, \mathrm{HC}=\right) ; 5.13(d, \mathrm{~J}=5.8$, $\left.\mathrm{MeCO}_{2} \mathrm{CH}\right) ; 4.50,4.45\left(A B, J=12.1, \mathrm{PhCH}_{2} \mathrm{O}\right) ; 3.40\left(\mathrm{~s}, \mathrm{SiCH}_{2} \mathrm{O}\right) ; 2.02\left(\mathrm{~s}, \mathrm{MeCO}_{2}\right) ; 1.97-1.78(\mathrm{~m}$, $\left.\mathrm{Me}_{2} \mathrm{CH}\right) ; 1.80(d, J=7.4, \mathrm{MeHC}=) ; 0.96(s, t-\mathrm{Bu}) ; 0.84,0.83\left(2 d, J=6.8,6.7, \mathrm{Me} e_{2} \mathrm{CH}\right) ; 0.23(s$, MeSi). ${ }^{3} \mathrm{C}$ NMR: $170.2(s, \mathrm{C}=0) ; 141.0$ ( $\left.d, \mathrm{HC}=\right) ; 138.9$ (s, SiC=); $135.2(s$, arom. C); 128.0, 127.3 (2d, each 2 arom. C); 127.1 (d, arom. C); $82.3\left(d, \mathrm{MeCO}_{2} \mathrm{CH}\right) ; 77.0\left(t, \mathrm{PhCH}_{2} \mathrm{O}\right) ; 62.1\left(t, \mathrm{SiCH}_{2} \mathrm{O}\right)$; $31.6\left(d, \mathrm{Me}_{2} \mathrm{CH}\right) ; 27.8\left(q, \mathrm{Me}_{3} \mathrm{C}\right) ; 21.2\left(q, \mathrm{MeCO}_{2}\right) ; 20.1$ (q, MeHC=); $19.0(q, \mathrm{MeCH}) ; 18.8(s$, $\left.\mathrm{Me}_{3} \mathrm{C}\right) ; 16.4(q, \mathrm{MeCH}) ;-6.2(q, \mathrm{MeSi}) . \mathrm{Cl}-\mathrm{MS}: 394\left(10,\left[\mathrm{M}+\mathrm{NH}_{4}\right]^{+}\right), 238(100)$.

5. Preparation of the Allenes. - 5.1. General Procedure: To solns. of the corresponding silylated allylic acetates (15a-15m or 16a-16c) in dried DMSO (approx. 0.05-0.10M) was added CsF (4 eq.). It was stirred at the respective temperatures (see Table 2) until full conversion of the starting esters (TLC evidence). The solns. were cooled to $0^{\circ} \mathrm{C}$ and $\mathrm{H}_{2} \mathrm{O}$ (approx. $20 \mathrm{ml}$ ) was added. The aqueous solns. were extracted with $\mathrm{Et}_{2} \mathrm{O}$, the combined organic phases dried over $\mathrm{MgSO}_{4}$, and carefully concentrated to $1 \mathrm{ml}$. Chromatography (hexane) of the residues gave the corresponding allenes (exact conditions see Table 2).

5.1. 1-Phenylbuta-1.2-diene (17a). ${ }^{1} \mathrm{H}$ NMR: 7.36-7.29 (m, 5 arom. $\left.H\right) ; 6.10(d q, J=6.4$, 3.1, $\mathrm{PhHC}=) ; 5.55(d q, J=6.4,7.2, \mathrm{MeHC}=) ; 1.80(d d, J=7.2,3.1, \mathrm{Me}) .{ }^{13} \mathrm{C}$ NMR: $205.9(s,=\mathrm{C}=)$; 134.9 (s, arom. C); 128.4 (d, 2 arom. C) 126.5 (d, 3 arom. C); 93.9 (d, PhHC=); 89.4 (d, MeHC=); $14.0(q, \mathrm{Me})$.

5.2. 1-Phenylpenta-1,2-diene (17b). IR: $3100 \mathrm{w}, 3080 \mathrm{w}, 3060 \mathrm{~m}, 3030 \mathrm{~m}, 2960 \mathrm{~s}, 2930 \mathrm{~s}$, $2870 m, 2850 m, 1945 s, 1800 w, 1750 w, 1705 w, 1675 w, 1600 m, 1575 w, 1495 s, 1455 s, 1405 w$, $1375 w, 1330 m, 1305 w, 1295 w, 1265 w, 1240 w, 1195 w, 1175 w, 1155 w, 1115 w, 1070 m, 1025 m$, $910 \mathrm{~m}, 875 \mathrm{~s}, 800 \mathrm{w}, 790 \mathrm{~m}, 760 \mathrm{~s}, 720 \mathrm{~m}, 700 \mathrm{~s}, 690 \mathrm{~s}$. ${ }^{1} \mathrm{H}$ NMR: $7.31-7.15$ ( $\left.\mathrm{m}, 5 \mathrm{arom} . \mathrm{C}\right) ; 6.16$ (dt, $J$ $=6.3,3.2, \mathrm{PhHC}=) ; 5.64\left(q, J=6.3, \mathrm{CH}_{2} \mathrm{HC}=\right) ; 2.16\left(d d q, J=6.7,3.2,7.4, \mathrm{MeCH}_{2}\right) ; 1.09(t, \mathrm{~J}=$ 7.4, $\left.\mathrm{MeCH}_{2}\right) .{ }^{13} \mathrm{C}$ NMR: 204.8. ( $\left.s,=\mathrm{C}=\right) ; 135.1$ (s, arom. C); 128.5, 126.6 (2d, each 2 arom. $\mathrm{C}$ ); 126.5 (d, arom. C); 96.7 (d, $\mathrm{PhHC}=) ; 95.1(d, \mathrm{MeHC}=) ; 21.9\left(t, \mathrm{MeCH}_{2}\right) ; 13.4\left(t, \mathrm{MeCH}_{2}\right)$. Cl-MS: $145\left([\mathrm{M}+\mathrm{H}]^{+}\right)$.

5.3. 1,3-Diphenylallene: (17c). IR $\left(\mathrm{CH}_{2} \mathrm{Cl}_{2}\right): 3070 \mathrm{~s}, 3060 \mathrm{~s}, 3020 \mathrm{~s}, 3000 \mathrm{~s}, 2950 \mathrm{~m}, 2750 \mathrm{w}$, $1935 s, 1895 w, 1880 w, 1825 w, 1805 w, 1750 w, 1700 w, 1670 w, 1595 s, 1490 s, 1450 s, 1330 w$, $1320 w, 1305 w, 1255 s, 1200 m, 1095 s, 1070 s, 1020 s, 1010 s, 910 s, 875 s .{ }^{1} H$ NMR: $7.45-6.91$ $(m, 10$ arom. $C) ; 6.62(s, 2$ PhHC $=)$. ${ }^{13} \mathrm{C}$ NMR: $207.8(s,=C=) ; 133.6(s, 2$ arom. $C) ; 128.9,127.3$ (2d, each 2 arom. C); 127.0 (d, 2 arom. C); 98.4 (d, 2 PhHC=). Cl-MS: $123\left([M+H]^{+}\right)$.

5.4. 4-Methyl-1-phenyl-1,2-pentadiene (17d). IR ( $\left.\mathrm{CHCl}_{3}\right)$ : 2950s, 2920s, 2870s, 2860s, $1945 \mathrm{~m}, 1600 \mathrm{~m}, 1490 \mathrm{~m}, 1455 \mathrm{~m}, 1375 \mathrm{~m}, 1360 \mathrm{w}, 1295 \mathrm{w}, 1250 \mathrm{w}, 1160 \mathrm{~m}, 1120 \mathrm{~m}, 1070 \mathrm{~m}, 1000 \mathrm{w}$ $910 \mathrm{w}, 875 \mathrm{~m} .{ }^{1} \mathrm{H}$ NMR: $7.43-7.14(\mathrm{~m}, 5 \mathrm{arom} . \mathrm{H}) ; 6.17(d d, J=6.4,3.0, \mathrm{PhHC}=) ; 5.59(t, J=6.1$, i$\mathrm{Pr} H \mathrm{C}=) ; 2.47-2.42\left(\mathrm{~m}, \mathrm{Me}_{2} \mathrm{CH}\right) ; 1.10,1.09(2 d, J=6.8,6.7, \mathrm{Me} 2 \mathrm{CH})$.

5.5. 1,6-Diphenyl-1,2-pentadiene (17e). IR: $3100 w, 3080 \mathrm{~m}, 3060 \mathrm{~s}, 3030 \mathrm{~s}, 2820 \mathrm{~s}, 2750 \mathrm{~m}$, $1950 s, 1875 w, 1805 w, 1745 w, 1700 w, 1600 m, 1495 s, 1450 s, 1435 w, 1405 w, 1380 w, 1335 w$, $1310 w, 1295 w, 1265 w, 1245 w, 1195 w, 1175 w, 1155 w, 1070 m, 1025 m, 1000 w, 910 m, 875 s$, $840 w, 775 s, 760 s, 745 s, 730 s, 720 s, 700 s$. ${ }^{1} \mathrm{H}$ NMR: $7.39-7.24$ ( $m, 10$ arom. C); 6.21 (dt, $J=6.6$, 2.9, $\mathrm{PhHC}=) ; 5.68\left(q, J=6.6, \mathrm{CH}_{2} \mathrm{HC}=\right) ; 2.84-2.74\left(m, \mathrm{CH}_{2} \mathrm{HC}=\right) ; 2.51-2.43\left(m, \mathrm{PhCH}_{2}\right) .{ }^{13} \mathrm{C}$ NMR: $205.2(s,=C=) ; 141.5$ ( $s$, arom. $C$ ); 134.8 ( $s$, arom $C$ ); 128.5, 128.4 (2d, each 2 arom. $C$ ); 128.3 (d, arom. C); 126.6, 126.5 (2d, each 2 arom. C); 125.9 (d, arom. C); 94.9 (d, PhHC=); 94.3 (d, $\left.\mathrm{CH}_{2} \mathrm{HC}=\right) ; 35.3\left(t, \mathrm{CH}_{2} \mathrm{CH}\right) ; 30.5\left(t, \mathrm{PhCH}_{2}\right)$. Cl-MS: $221\left([\mathrm{M}+\mathrm{H}]^{+}\right)$.

6. Synthesis of the Optically Active Compounds. $-6.1 .(R)-(-)-[($ Benzyloxymethyl)(tert-butyl)methylsilyl] Ethyl Ketone (-)-13 and (R)- $(+)-[($ Benzyloxymethyl)(tert-butyl)methylsilyl] Prop-2-enyl Ether. To a freshly prepared solution of LDA $(1.5 \mathrm{mmol})$ in THF $(5 \mathrm{ml})$ was added $(R)-(-)$ [(benzyloxy)methyl](tert-butyl)methylsilyl methyl ketone $[(-)-12](0.30 \mathrm{mg}, 1.14 \mathrm{mmol})$ at $-80^{\circ} \mathrm{C}$. After $1 \mathrm{~h}, \mathrm{DMPU}(0.85 \mathrm{ml}, 7 \mathrm{mmol})$ was added. It was stirred for an additional $30 \mathrm{~min}$ and treated with methyl iodide $(0.48 \mathrm{~g}, 3.38 \mathrm{mmol})$. The temperature was increased gradually to $23^{\circ} \mathrm{C}(30 \mathrm{~min})$, then it was quenched with a saturated aqueous $\mathrm{NH}_{4} \mathrm{Cl}$ solution The aqueous layer was extracted with $\mathrm{Et}_{2} \mathrm{O}$, and the combined organic phases were dried over $\mathrm{Mg}_{2} \mathrm{SO}_{4}$ and evaporated. The crude product gave after chromatography (hexane/ethyl acetate $35: 1)(-)-13(0.22 \mathrm{~g}, 0.79 \mathrm{mmol}, 70 \%)$ 
and $(R)-\{($ (benzyloxy)methyl $]$ (tert-butyl)methylsilyl\} propen-2-yl ether $(50 \mathrm{mg}, 0.18 \mathrm{mmol}, 16 \%)$ as colorless oils.

Data of $(-)-13:[\alpha]_{D}^{23}=-10.9 \pm 2$ (c=0.8, THF). IR: $3080 w, 3060 w, 3025 w, 2959 s, 2925 s$, $2880 \mathrm{~s}, 2850 \mathrm{~m}, 2810 \mathrm{~m}, 1640 \mathrm{~s}, 1495 \mathrm{w}, 1460 \mathrm{~m}, 1430 \mathrm{w}, 1400 \mathrm{w}, 1390 \mathrm{w}, 1375 \mathrm{~m}, 1360 \mathrm{~m}, 1320 \mathrm{~s}$, $1250 m, 1200 w, 1095 s, 1070 s, 1025 m, 1010 w, 980 w, 940 w, 900 w, 825 s, 805 m, 775 s, 735 s$, 595s. ${ }^{1} \mathrm{H}$ NMR: $7.38-7.28\left(m, 5\right.$ arom.H); $4.50\left(s, \mathrm{PhCH}_{2} \mathrm{O}\right) ; 3.44,3.38\left(A B, J=13.0, \mathrm{SICH}_{2} \mathrm{O}\right)$; $2.67\left(q, J=7.2, \mathrm{MeCH}_{2}\right) ; 0.96(s, t-\mathrm{Bu}) ; 0.95\left(t, \mathrm{MeCH}_{2}\right) ; 0.24$ (s, MeSi). ${ }^{13} \mathrm{C}$ NMR: 138.4 (s, arom. C); 128.3, $127.6\left(2 d\right.$, each 2 arom. C); $77.3\left(t, \mathrm{PhCH}_{2} \mathrm{O}\right) ; 59.4\left(t, \mathrm{SiCH}_{2} \mathrm{O}\right) ; 44.0\left(t, \mathrm{MeCH}_{2}\right) ; 26.8$ (q, Mes $\mathrm{C}) ; 16.7$ (s, $\left.\mathrm{Me}_{3} C\right) ; 5.8\left(q, \mathrm{MeCH}_{2}\right) ;-9.7$ (q, MeSi). Cl-MS; $279\left([M+\mathrm{H}]^{+}\right)$.

Data of $(R)-(+)-\left\{\left((B e n z y l o x y)\right.\right.$ methyl](tert-butyl)methylsilyl]\} Propen-2-yl Ether. $[\alpha]_{D}^{23}=19.9 \pm 2$ (c = 0.7, THF). IR: $3080 w, 3060 w, 3020 w, 2990 w, 2959 s, 2939 s, 2890 m, 2850 s, 1575 s, 1495 w$, $1460 \mathrm{~m}, 1435 \mathrm{w}, 1390 \mathrm{w}, 1375 \mathrm{~m}, 1360 \mathrm{~m}, 1250 \mathrm{~m}, 1215 \mathrm{~s}, 1090 \mathrm{~s}, 1070 \mathrm{~s}, 1035 \mathrm{~s}, 1010 \mathrm{w}, 980 \mathrm{w}$, $935 w, 895 s, 825 s, 785 s, 775 s, 735 s, 695 s, 675 w .{ }^{1} \mathrm{H}$ NMR: $7.38-7.25$ ( $m, 5$ arom. H); $4.72(d, J$ $=2.2, \mathrm{HC}=) ; 4.50$ (s, $\mathrm{PhCH} \mathrm{H}_{2} \mathrm{O}$ ); $4.38(d, J=2.2, \mathrm{HC}=) ; 3.51$ (s, MeC=); 3.39, 3.32 ( $A B, J=13.0$, $\mathrm{SiCH}_{2} \mathrm{O}$ ); 0.95 (s, t-Bu); 0.17 (s, MeSi). ${ }^{13} \mathrm{C}$ NMR: 167.1 (s, MeC=); 138.9 (s, arom. C); 128.1, 127.3 (2d, each 2 arom. C); $95.7\left(t, \mathrm{H}_{2} \mathrm{C}=\right) ; 76.9\left(t, \mathrm{PhCH}_{2} \mathrm{O}\right) ; 59.9\left(t, \mathrm{SiCH}_{2} \mathrm{O}\right) ; 53.7(q, \mathrm{MeC}=)$; $26.9\left(q, \mathrm{Me}_{3} \mathrm{C}\right) ; 16.5\left(s, \mathrm{Me}_{3} \mathrm{C}\right) ;-9.4$ (q, MeSi). Cl-MS: $279\left([\mathrm{M}+\mathrm{H}]^{+}\right)$.

6.2. (-)-(E)-2-\{[(Benzyloxy)methyl](tert-butyl)methylsilyl \}-1-phenylbut-2-en-1-ol ((-)-11c and $(-)-11 \mathrm{~d})$. To a solution of $(-)-13(0.18 \mathrm{~g}, 0.65 \mathrm{mmol})$ in $\mathrm{MeCN}(5 \mathrm{ml})$ was added 2,4,6-triisopropylphenylsulfonylhydrazide $(0.23 \mathrm{~g}, 0.77 \mathrm{mmol})$ at $23^{\circ} \mathrm{C}$. It was stirred for $24 \mathrm{~h}$ and the solvent evaporated to give after chromatography (hexane/ethylacetate 25:1) the corresponding hydrazone $(0.36$ $\mathrm{g}, 0.64 \mathrm{mmol}, 98 \%)$ as a colorless oil. $[\alpha]_{D}^{23}=-23 \pm 2\left(c=0.9\right.$, THF). ${ }^{1} \mathrm{H}$ NMR: $8.0(s, N H) ; 7.41-7.23$ $\left(m, 5\right.$ arom. H); $7.14\left(s, 2\right.$ arom. H); $4.37\left(s, \mathrm{PhCH}_{2} \mathrm{O}\right.$ ); 4.17 (hept., $\left.J=6.7,2 \mathrm{Me}_{2} \mathrm{CH}\right) ; 3.11(\mathrm{~s}$, $\mathrm{SiCH}_{2} \mathrm{O}$ ); 2.88 (hept., $\left.J=6.9, \mathrm{Me}_{2} \mathrm{CH}\right) ; 2.88\left(q, J=7.6, \mathrm{MeCH}_{2}\right) ; 1.27-1.19\left(\mathrm{~m}, 18 \mathrm{H}, 3 \mathrm{Me}_{2} \mathrm{CH}\right.$ ); $1.01\left(t, J=6.7, \mathrm{MeCH}_{2}\right) ; 0.69(s, t-\mathrm{Bu}) ; 0.07$ (s, MeSi). ${ }^{13} \mathrm{C} \mathrm{NMR:} 166.7$ (s, C=N); 153.1 (s, arom. C); 151.0 (s, 2 arom. C); 138.5 (s, arom. C); 132.0 (s, arom. C); 128.6, 128.1 (2d, each 2 arom. $C$ ); 127.4 (d, arom. C); 77.1 ( $\left.t, \mathrm{PhCH}_{2} \mathrm{O}\right) ; 60.1\left(t, \mathrm{SiCH}_{2} \mathrm{O}\right) ; 34.1$ (d, $\left.\mathrm{Me}_{2} \mathrm{CH}\right) ; 29.7\left(d, 2 \mathrm{Me}_{2} \mathrm{CH}\right) ; 26.6$ $\left(q, 4 \mathrm{Me}_{2} \mathrm{CH}\right) ; 24.8\left(q, \mathrm{Me}_{3} \mathrm{C}\right) ; 23.5\left(q, \mathrm{Me}_{2} \mathrm{CH}\right) ; 23.0\left(t, \mathrm{MeCH}_{2}\right) ; 17.0\left(s, \mathrm{Me}_{3} \mathrm{C}\right) ; 8.9$ (q, $\left.\mathrm{MeCH}_{2}\right) ;$ -9.3 (q, MeSi). Cl-MS: $559[M+\mathrm{H}]^{+}$

This hydrazone $(210 \mathrm{mg}, 0.37 \mathrm{mmol})$ was dissolved in hexane/Et $2 \mathrm{O}(1: 1,6 \mathrm{ml})$, and sec-BuLi $\left(0.9 \mathrm{ml}, 1.3 \mathrm{M}\right.$ in pentane) was added at $-80^{\circ} \mathrm{C}$. It was stirred for $4 \mathrm{~h}$, then the temperature was slowly raised to $-10^{\circ} \mathrm{C}(10 \mathrm{~min})$, stirring continued for an additional $15 \mathrm{~min}$, and quenched with benzaldehyde $(0.1 \mathrm{ml}, 0.82 \mathrm{mmol})$. Saturated aqueous $\mathrm{NH}_{4} \mathrm{Cl}$ solution was added, the aqueous layer extracted with $\mathrm{Et}_{2} \mathrm{O}$, and the combined organic phases were dried over $\mathrm{MgSO}_{4}$ and evaporated. The crude product gave after chromatography (hexane/ethyl acetate $25: 1)(-)-11 \mathrm{c}(65 \mathrm{mg}, 0.18 \mathrm{mmol}$ ) and $(-)-11 \mathrm{~d}$ ( $65 \mathrm{mg}, 0.18 \mathrm{mmol}$ ) as colorless oils (overall $130 \mathrm{mg}, 0.35 \mathrm{mmol}, 95 \%)$. Characterization see 3.9 .

\section{References}

(1) "Chemistry of Allenes", Ed.: Landor SR, Academic Press, London, 1982, vol. 1-3.

(2) Chan TH, and Mychajlowskij W, Tetrahedron Lett., 15 (1974), 171.

(3) Chan TH, Mychailowskij W, Ong BS, and Harpp DN, J. Org. Chem., 43 (1978), 1526.

(4) Gröbel B-T, and Seebach D, Chem. Ber., 110 (1977), 867.

(5) Chan TH, and Mychajlowskij W, Tetrahedron Lett., 15 (1974), 3479.

(6) Torres E, Larson GL, and McGarvey GJ, Tetrahedron Lett., 29 (1988), 1355.

(7) Enev V, Stojanova D, and Bienz S, Helv. Chim. Acta, 79 (1996), 391.

(8) Wilson SR, and Jacob.LA, J. Org. Chem., 51 (1986), 4833.

(9) Bratovanov S, Ph.D. thesis, University of Zurich, in preparation.

(10) Zweifel G, Murray RE, and On HP, J. Org. Chem., 46 (1981), 1292.

(11) Negishi E, and Takahashi T, J. Am. Chem. Soc., 108 (1986), 3402.

(12) Huber P, Bratovanov S, Bienz S, Syldatk C, and Pietzsch M, Tetrahedron: Asymm., 7 (1996), 69.

\section{Received: August 6, 1996 - Accepted: August 14, 1996 - Received in revised camera-ready format: August 16, 1996}


Bereitgestellt von | UZH Hauptbibliothek / Zentralbibliothek Zürich Angemeldet 